\title{
La formation du contrôle international des drogues
}

In: Déviance et société. 1999 - Vol. 23 - N4. pp. 395-419.

Citer ce document / Cite this document :

Dudouet François-Xavier. La formation du contrôle international des drogues. In: Déviance et société. 1999 - Vol. 23 - N4. pp. 395-419.

doi : $10.3406 /$ ds.1999.1704

http://www.persee.fr/web/revues/home/prescript/article/ds_0378-7931_1999_num_23_4_1704 


\section{Abstract}

This study has used the archives of the Society of Nations to reconsider the genesis of the international control of drugs during the period 1920-1930. It was during this period that the member states started to constrain the manufacture and international trade in drugs to medical usage, hence setting up a worldwide system of drug control which persists to today. The creation of this system involved a series of conventions which clearly distinguished legitimate and illegitimate uses of drugs. This distinction was made less at the level of the activity itself (manufacture, international trade) than in relation to those who were expressly permitted to undertake such activities. Hence the article proceeds to look at those who took part in creating the conventions and who, at the same time, were putting together a monopoly of manufacture and licit trade in drugs - states themselves and the large pharmaceutical companies.

\section{Zusammenfassung}

Auf der Basis von Archiven des Völkerbundes analysiert diese Untersuchung die Entstehung der internationalen Drogenkontrolle in den Jahren 1920-1930. In dieser Epoche haben sich die Staaten darauf geeinigt, die Herstellung und den internationalen Handel von Betäubungsmitteln auf medizinische Bedurfnisse zu begrenzen und so ein System der weltweiten Drogenkontrolle etabliert, das auch heute noch besteht. Die Entstehung dieses Systems manifestiert sich in einer Serie von Vereinbarungen, in denen der erlaubte und der unerlaubte Gebrauch von Drogen festgelegt worden ist. Diese Unterscheidung zwischen erlaubtem und unerlaubtem Gebrauch betrifft weniger den Gebrauch selbst (Herstellung, internationaler Handel), als vielmehr die Akteure, denen explizit der Gebrauch erlaubt ist. Deshalb bezieht sich der Artikel auf diese Akteure, die an der Ausarbeitung der Vereinbarungen beteiligt waren und die zur damaligen Zeit ein Monopol der Herstellung und des legalen Handels mit Betäubungs-mitteln hatten: im wesentlichen die Staaten und große pharmazeutische Unternehmen.

Tot stand gekomen op basis van de archieven van de Volkenbond, biedt deze studie aan om terug te komen op de genèse van de internationale contrôle inzake drugs in de twintiger en der- tiger jaren. Het is op dat ogenblik dat de diverse Staten mekaar vonden om de fabricatie en de internationale handel voor medische doeleinden te beperken, en dit door de installatie van een mondiaal beheerssysteem dat momenteel nog steeds in werking is. De indeplaatsstelling van dit systeem wordt geuit door een geheel van conventies die het legaal en illegaal gebruik van drugs duidelijk onderscheiden. Dit onderscheid doet zich minder voor bij de gebruikers zelf (fabricatie, internationale handel) dan wel bij de actoren die uitdrukkellijk toegelaten worden om ze te ontplooien. Dat is de reden waarom het artikel terug wil komen op de actoren die deel- namen aan de totstandkoming van de internationale conventies en die, terzelfdertijd, zich het monopolie van de fabricatie en de légale handel van drugs toeëigenden, met name de Staten en de grote pharmaceutische groepen.

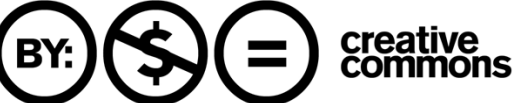




\title{
LA FORMATION DU CONTRÔLE INTERNATIONAL DES DROGUES
}

\author{
F.-X. DUDOUET*
}

\begin{abstract}
Etablie sur la base des archives de la Société des Nations, cette étude se propose de revenir sur la genèse du contrôle international des drogues dans les années 1920-1930. C'est à cette époque que les Etats se sont entendus pour limiter la fabrication et le commerce international des stupéfiants aux seuls besoins médicaux, instaurant ainsi un système de gestion mondial des drogues encore à l'œuvre de nos jours. La mise en place de ce système s'objective à travers une série de conventions qui départagent clairement les usages légitimes des stupéfiants des usages illégitimes. Cette distinction entre usages légitimes et usages illégitimes se fait moins à partir des usages eux-mêmes (fabrication, commerce international) que par les acteurs qui sont expressément autorisés à les exercer. C'est pourquoi l'article revient sur ces acteurs qui ont pris part à l'élaboration des conventions internationales et qui, dans le même temps, se sont octroyés le monopole de la fabrication et du commerce licite des stupéfiants, à savoir pour l'essentiel les Etats et les grands groupes pharmaceutiques.
\end{abstract}

Mots-ClÉS: DROGUES - CONTRÔLE INTERNATIONAL DES DROGUES - HistoIRE DES DROGUES RELATIONS INTERNATIONALES - FABRICATION ET COMMERCE INTERNATIONAL DES STUPÉFIANTS

En juillet 1931, la Convention qui vient d'être adoptée sur la limitation de la fabrication des stupéfiants est un précédent alors sans équivalent dans les relations internationales. Pour la première fois, un espace économique et sanitaire fonctionnant suivant les mêmes règles pour tous les Etats du monde vient d'être créé. A compter de 1933, date de l'entrée en vigueur de la Convention, toute entreprise ou tout individu fabricant des stupéfiants sans l'autorisation officielle de l'Etat où ils résident sont considérés comme hors la loi. Tous les fabricants' de stupéfiants sont dorénavant référencés et leurs productions surveillées. Mieux encore la fabrication mondiale de stupéfiants est strictement limitée aux seuls besoins médicaux et scientifiques. Un mode de régulation international contrôle, de la fabrication à la prescription médicale en passant par le commerce international, les usages légitimes des stupéfiants. Les activités illégales apparaissent alors a contrario de l'établissement de ces usages légitimes. En ce sens le contrôle international des drogues (CID) est avant tout l'institutionnalisation d'une distinction entre des usages légitimes et des usages illégitimes. La distinction entre ces usages permet de penser que

* Laboratoire d'analyse des systèmes politiques (CNRS), Université Paris X Nanterre.

1 Nous utilisons le terme de «fabricant de stupéfiants " plutôt que celui d' « industrie pharmaceutique », car, même si les deux types d'activité sont souvent présentes au sein d'une même entreprise, elles ne sont pas identiques l'une à l'autre. 
le processus de construction du contrôle international des drogues repose d'abord sur un travail d'objectivation ${ }^{2}$ du problème des drogues. C'est-à-dire, sur la formation intellectuelle d'une frontière nette entre l'espace des usages autorisés et celui des usages condamnés. On parle aujourd'hui du problème des drogues en utilisant un ensemble de représentations solidement établies qui servent de repères aux débats et donnent sens aux diverses positions. Cela n'a pas toujours été le cas. Les termes du débat, la désignation des enjeux ne se sont pas imposés d'emblée, tout cela a été construit au début du siècle, notamment sous l'égide de la Société des Nations, par l'entremise et la confrontation de stratégies différenciées. En effet, le choix des enjeux, la mise en évidence de tel ou tel aspect ne sont pas fortuits et ne s'imposent pas sous le mode de l'évidence. Ainsi, les premiers efforts du contrôle international des drogues se consacrèrent plus particulièrement au contrôle de la fabrication pharmaceutique des stupéfiants. Alors que la plupart des pays occidentaux avaient édicté des lois qui limitaient la consommation légitime des stupéfiants aux seuls besoins médicaux et scientifiques, les fabricants, eux, n'étaient soumis à aucune restriction. Ce sont leurs produits que l'on retrouvait d'ailleurs chez les consommateurs illégitimes. Il existait, certes, un enjeu sanitaire dans la limitation des stupéfiants manufacturés, mais pourquoi la Commission consultative du trafic de l'opium de la Société des Nations (CCO) a-t-elle consacré la plupart de ses travaux à cette question, plutôt qu'à celle de la production d'opium ou de la consommation de l'opium fumé en Asie?

Si la question des stupéfiants manufacturés s'est imposée comme l'un des enjeux majeurs du problème des drogues durant l'entre deux guerres, c'est parce que certains acteurs ont réussi à le construire et à l'imposer comme problème primordial. D'autres représentations du problème des drogues étaient en concurrence avec celle concernant la limitation de la fabrication pharmaceutique, pourtant elles ont été placées au second plan ou traitées bien plus $\operatorname{tard}^{3}$. Le fait que certaines représentations aient pris le pas sur d'autres invite à penser qu'il existait un rapport de force sous-jacent à leur mise en forme. Pour bien comprendre la construction des objectivations qui déterminèrent la formation du CID, il est nécessaire de supposer l'existence d'un espace relationnel spécifique, lui-même en pleine construction, engageant des acteurs aux intérêts et aux stratégies différenciés. L'existence de cet espace relationnel conduit à considérer le contrôle international des drogues comme étant avant tout cet espace relationnel, ou plus exactement une configuration sociale ${ }^{4}$. Objectivation et configuration sont inséparables l'une de l'autre. Les représentations participent à la définition des relations sociales, des positions occupées par chacun. Les rapports de force déterminent quant à eux l'univers du pensable et du possible, les enjeux importants de ceux qui sont secondaires.

C'est en suivant cette double grille de lecture qui engage représentations et rapports de force que nous tenterons d'expliquer comment s'est construit le contrôle international des drogues sur un point particulier qui est la limitation de la fabrication des stupéfiants. La période étudiée s'étend entre 1921, date de la première session de la Commission du trafic de l'opium de la Société des Nations, et 1931, année de l'adoption de la Convention pour la limitation de la fabrication. Durant ces dix années, le Contrôle international des drogues passe d'un espace faiblement structuré à une configuration fortement organisée, qui impose à l'ensemble de la planète une économie dirigée des stupéfiants.

2 Le terme d'objectivation est à entendre ici dans un sens proche de celui développé par Lacroix, 1985. On verra aussi dans le même esprit Bourdieu, 1976. Cependant, l'objectivation concerne ici aussi bien la mise en forme des représentations des rapports sociaux que de l'enjeu autour et pour lequel ils s'organisent: celui du problème des drogues.

3 C'est le cas notamment pour la culture des stupéfiants. Certains comme l'URSS considéraient que l'on ne pouvait limiter la fabrication des stupéfiants sans en limiter la source: la culture de l'opium et de la feuille de coca.

$4 \quad$ Nous utilisons le terme de configuration sociale dans le sens où l'entend et l'a défini Elias, 1991. 
Si la configuration globale du contrôle international des drogues doit être envisagée dans ses dimensions les plus larges, c'est-à-dire en incluant les consommateurs comme les membres des institutions internationales, on peut néanmoins repérer un nombre restreint d'acteurs qui ont joué un rôle clef dans la mise en place du contrôle de la fabrication des stupéfiants. La plupart de ces acteurs étaient membres de la Commission consultative du trafic de l'opium. C'est pourquoi, cette étude se concentrera plus particulièrement sur cette institution.

Après avoir situé la Commission au sein de la configuration globale et dans son contexte institutionnel, nous examinerons comment certains de ses membres en relations avec d'autres espaces sont parvenus à édifier les bases du contrôle international des drogues.

\section{La configuration du contrôle international des drogues}

\section{La configuration globale}

Nous appelons configuration globale du contrôle international des drogues l'espace relationnel formé par tous les individus participant à un titre ou à un autre à la question des stupéfiants. C'est pourquoi, il nous faut dans cet espace spécifique considérer aussi bien le consommateur de stupéfiants (que ce soit un consommateur légitime ou illégitime) que les représentants des Etats intervenant aux plus hauts niveaux. Afin de mieux représenter les relations d'interdépendances complexes qui lient les différents acteurs de la configuration, nous proposons un schéma (Schéma I) représentant les types d'acteurs que l'on pouvait rencontrer à différents niveaux entre 1920 et 1940 sur la question spécifique des stupéfiants manufacturés. L'objectivation que nous présentons ici n'est qu'un modèle d'analyse, une grille de lecture des phénomènes sociaux et non une représentation définitive du contrôle international des drogues.

Les types d'acteurs identifiés ne sont pas seulement de notre fait, la plupart d'entre eux sont désignés par les membres de la Commission consultative du trafic de l'opium de la SDN. Ces derniers identifient clairement certains types d'acteurs qui leur permettent d'organiser et de donner sens à l'univers des drogues. Ainsi, il est aisé de repérer dans les rapports et les procès verbaux de la Commission: les fabricants de stupéfiants, les gouvernements compris

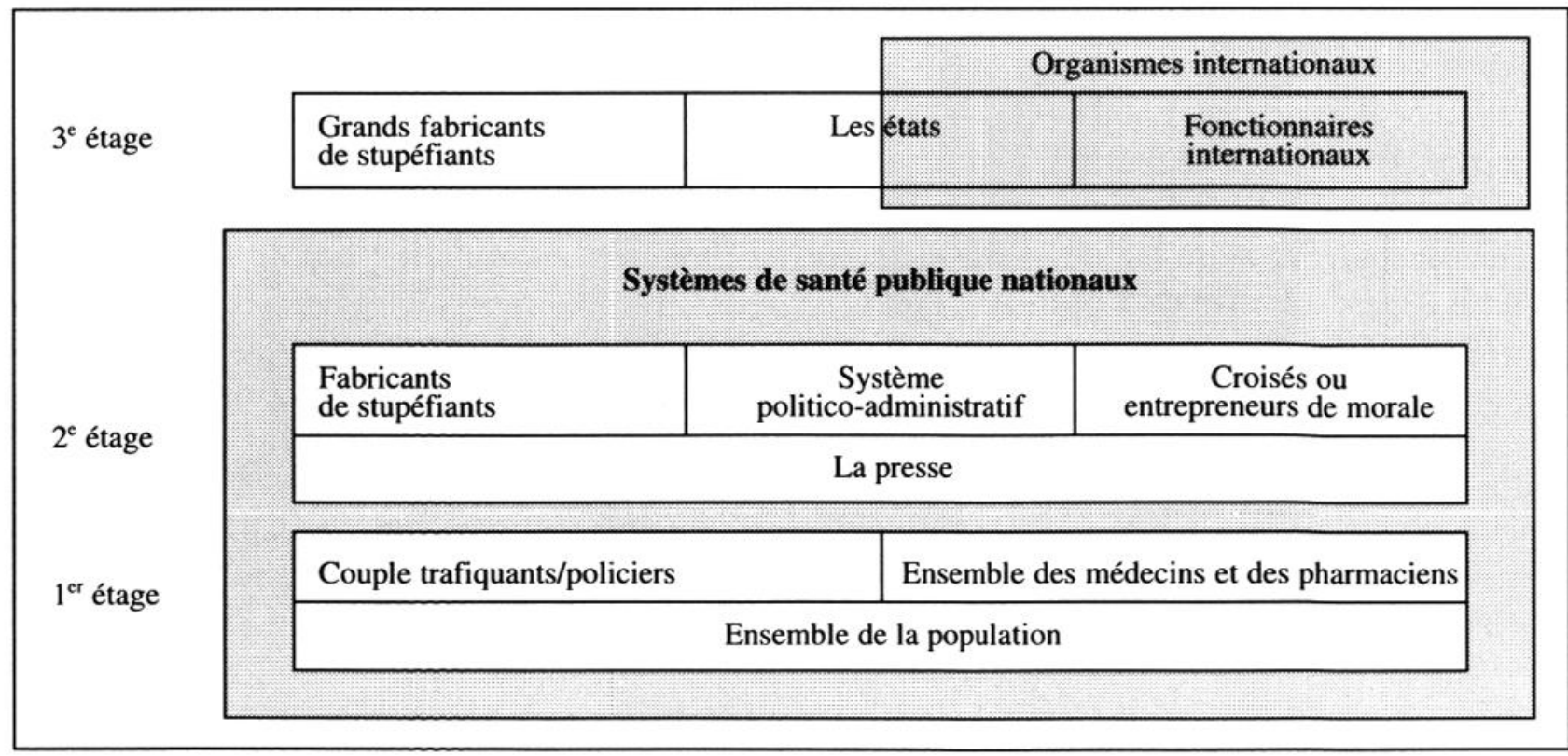

Schéma I: Types d'acteur entrant dans la configuration du contrôle international des drogues. 
comme l'appareil de domination nationale, la presse citée telle quelle et entendue comme l'intermédiaire entre la Commission et l'opinion publique, les trafiquants et les policiers ${ }^{5}$, enfin on trouve aussi les institutions internationales et les fonctionnaires désignés généralement sous le couvert de l'institution qui les rassemble à savoir le Secrétariat de la Société des Nations. D'autres types d'acteurs ont été par contre construits par nous même en référence à d'autres travaux: les entrepreneurs de morale en filiation avec $\mathrm{H}$. Becker ${ }^{6}$, et l'ensemble des professions médicales comprises comme un groupe constitué en vue de garantir leur monopole sur la prescription et la délivrance, voire la gestion des stupéfiants ${ }^{7}$. Ces types d'acteurs sont en fait des outils de classification des individus agissant au sein du CID et une manière préalable de définir les relations entre une myriade d'acteurs individuels. La Commission consultative du trafic dc l'opium est donc à placer sur ce schéma dans la partie «Etats» du cadre «Organismes internationaux » du $3^{\mathrm{c}}$ étage.

La notion de type d'acteurs est moins à entendre comme une individualité abstraite et agissante que comme un espace particulier composé d'individus partageant des propriétés et parfois des représentations communes. A ce titre, chaque espace peut être entendu comme une sous configuration avec ses modes de relations d'interdépendances spécifiques. La CCO constitue elle-même une mini configuration. Les individus appartenant à un même espace peuvent être en concurrence entre eux, contracter s'ils en ont la possibilité des alliances avec des acteurs du même espace ou intervenant dans d'autres espaces. En outre, de nombreux individus, notamment ceux présents à l'étage international, relèvent de plusieurs espaces ${ }^{8}$.

\section{Le contexte institutionnel}

Avant de s'attacher plus particulièrement à la Commission consultative du trafic de l'opium et à ses membres, il est nécessaire de rappeler le contexte institutionnel dans lequel elle s'insère.

Le Schéma II présente les principales institutions spécialisées intervenant dans le contrôle international des drogues entre 1921 et 1940. L'Assemblée et le Conseil de la Société des Nations en tant qu'organes généraux n'ont pas été représentés. Les dates sous les sigles ou les noms sont celles où l'institution a effectivement commencé à fonctionner. Les institutions ont été regroupées suivant deux critères doubles. Les organes spécialisés dans les questions sanitaires et ceux consacrés spécifiquement aux drogues d'une part et les organes décisionnels et les organes d'expertise d'autre part. A l'exception de l'Office international d'hygiène publique tous sont des organes de la SDN. Enfin, les trois bandes coiffant le schéma représentent les différentes conventions spécifiant les fonctions de chaque institution.

Créée par l'Assemblée de la SDN lors de sa première session en 1920, la Commission consultative du trafic de l'opium est un organe de représentation intergouvernemental. Elle est chargée de veiller à la bonne application des conventions et de proposer au Conseil toutes mesures qu'elle jugera utile pour améliorer la bonne application des traités. Dans la pratique,

Sous avons réunis les policiers et les trafiquants en couple dans la mesure où ils sont dans une interaction quasi constante et que leurs comportements se définissent mutuellement.

6 Becker, 1985.

7 Voir entre autre sur ce sujet: Berridge V., Griffith E., 1987.

8 C'est le cas notamment de Sir Malcom Delevingne qui en tant que représentant du Royaume Uni au sein de la CCO appartient à l'espace des «organismes internationaux » mais aussi à celui des «Etats» du $3^{c}$ étage et en tant que fonctionnaire du «Home Office» à l'espace «Système politico-administratif». 


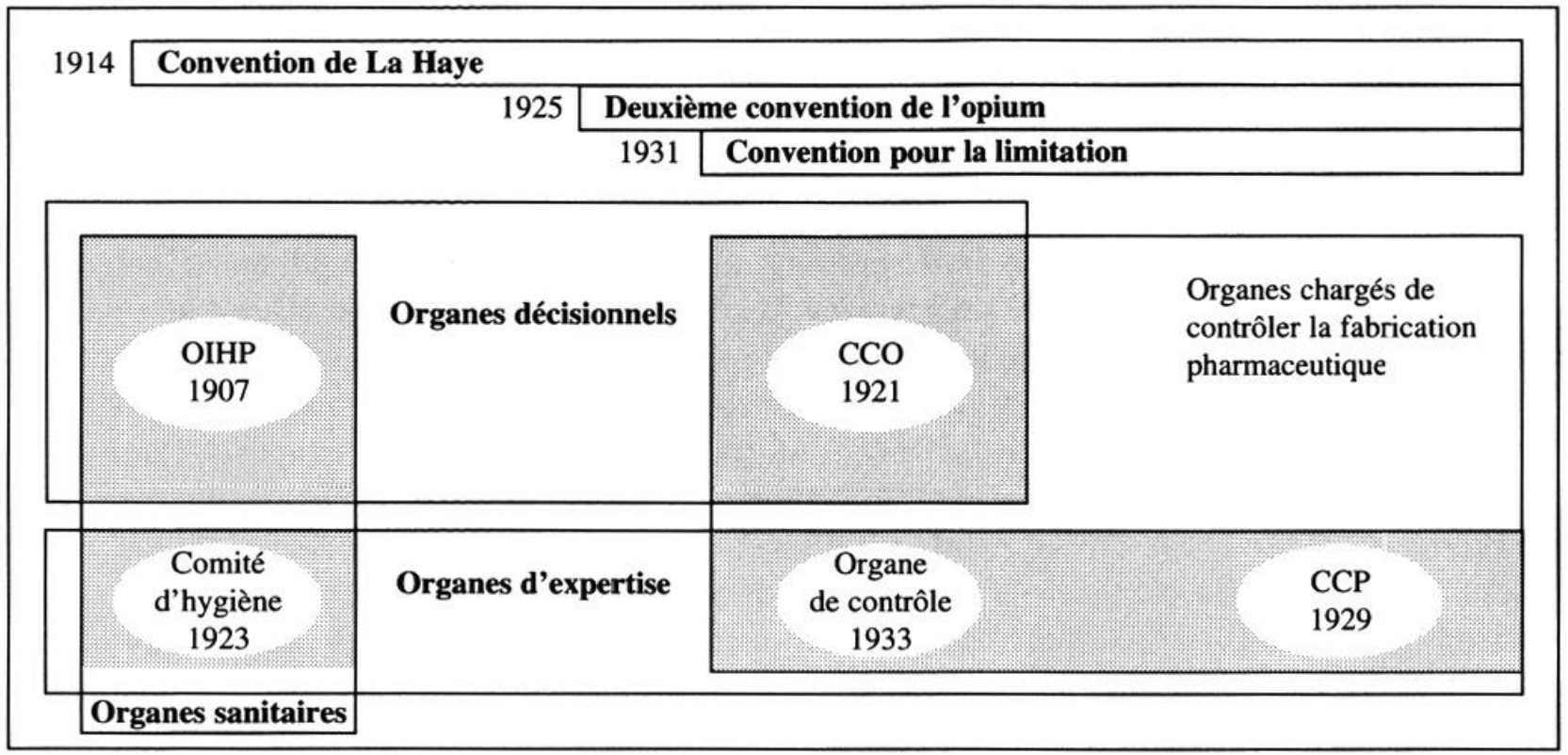

Schéma II: L'organisation institutionnelle du contrôle international des drogues 1920-1940.

elle constitue le véritable moteur du contrôle international des drogues. Elle désigne à partir de 1933 l'un des quatre membres de l'Organe de contrôle.

L'Office international d'hygiène publique (OIHP), crée avant la SDN, garde son autonomie. Il prend cependant en charge le Conseil général consultatif d'Hygiène de la Société des Nations et à ce titre discute des questions que peut lui soumettre le Comité d'hygiène publique de la SDN. Il désigne à partir de 1933 l'un des quatre membres de l'Organe de contrôle.

Le Comité d'hygiène est l'interlocuteur privilégié de la $\mathrm{CCO}$ pour toutes les questions médicales. Il est notamment consulté sur la nocivité de nouvelles substances en vue de leur indexation au régime du contrôle international des drogues. A partir de 1933, il désigne l'un des quatre membres de l'Organe de contrôle.

Le Comité central permanent de l'opium (CCP) créé par la Convention de 1925 est un organe d'expertise chargé de collecter les statistiques envoyées chaque année par les gouvernements sur les flux des stupéfiants. L'une de ses missions principales est de repérer les pays qui risquent de devenir des centres de trafic illicite. Ces membres sont des experts désignés par l'Assemblée. Il désigne à partir de 1933 l'un des quatre membres de l'Organé de contrôle.

L'Organe de contrôle mis en place par la Convention de 1931 sur la fabrication est chargé d'établir, à partir des déclarations des gouvernements' ${ }^{9}$, une évaluation de la consommation légitime pour toute la planète et de la fabrication mondiale nécessaire pour satisfaire ces besoins (dans les projets initiaux l'organe devait être une institution privée chargée de répartir chaque année entre les fabricants de stupéfiants la production mondiale ${ }^{10}$ ).

9 L'organe était aussi autorisé à établir ses propres évaluations à l'égard des Etats pour lesquels il n'aurait reçu aucune information, et que les Etats en question soient partie ou non à la Convention de 1931.

10 Voir sur ce point: SDN, Procès verbal de la Conférence sur la limitation de la fabrication des drogues nuisibles, quatrième séance, Assemblée préliminaire des pays manufacturiers des drogues, Londres, octobre 1930, Archives 12/23680/4003. 


\section{La Commission consultative du trafic de l'Opium}

\section{L'intérêt de la CCO comme objet d'étude}

La CCO se présente et est présentée par les acteurs du niveau international comme l'organe clef du contrôle international des drogues entre 1920 et 1940 . Cette représentation s'appuie sur plusieurs facteurs. Tout d'abord c'est le premier organe intergouvernemental spécialisé sur la question des drogues qui ait été créé. Malgré son statut d'organe consultatif et subordonné en cela au Conseil et à l'Assemblée de la Société des Nations, il s'impose très vite comme le véritable organe décisionnel (à l'exception des conférences intergouvernementales) sur la question des stupéfiants. Ceci est nettement visible par l'adoption quasi stricto-sensu de ses rapports, résolutions et recommandations par l'Assemblée et le Conseil de la SDN. De plus, toutes les Conventions adoptées durant la période sont d'abord élaborées en son sein. Même si les textes sont renégociés durant les conférences plénières, ils gardent l'empreinte des travaux préliminaires de la Commission. En ce sens la CCO oriente de manière significative le CID. D'autre part, en tant qu'organisation intergouvernementale, la CCO peut être considérée comme le cœur de la configuration formée par le troisième étage. On remarquera en effet que se manifestent en son sein entre 1921 et 1931 les différents types d'acteurs que nous avons repérés préalablement (notamment les entrepreneurs de morale, les policiers, les fabricants de stupéfiants, les fonctionnaires internationaux et bien sûr les Etats). D'une certaine manière, on peut concevoir la CCO comme le lieu où converge et se rencontre la plupart des intérêts concernant le contrôle international des drogues.

Il existe, en outre, une masse abondante de documents sur ses travaux qui facilite la recherche et donc son choix en tant qu'objet. Enfin, et c'est peut-être l'argument principal, la CCO est un lieu privilégié d'objectivation. Comme nous l'avons souligné préalablement les types d'acteurs que nous avons sélectionnés pour composer la configuration globale du CID ont d'abord été élaborés par les membres de la Commission. En tant que lieu de production des objectivations donnant sens à l'univers des drogues (et construisant à mesure de ces objectivations cet univers), la CCO mérite une attention particulière. Précisons tout de suite que la CCO n'est pas une sorte de Deus ex machina de production de sens, les objectivations qu'elle met en place viennent souvent d'autres espaces (médecin, fabricants de stupéfiants, entrepreneurs de morale). Cependant, même les représentations qui ne sont pas directement issues de ses travaux sont rediscutées et recontextualisées en fonction des enjeux qui lui sont propres (la question de la limitation des stupéfiants aux seules fins médicales et scientifiques, qui fut initiée par les professions médicales et les entrepreneurs de morale, est reconsidérée du point de vue de ses incidences économiques vis-à-vis des fabricants). Les représentations fixées par la CCO ont d'autant plus d'importance que ce sont elles qui seront discutées, adoptées ou rejetées par les conventions internationales, c'est à dire qui prendront éventuellement la force du droit. Nous touchons ici un point caractéristique du processus de construction du droit, et du droit international en particulier. A savoir que les textes adoptés sont une manière de fixer les rapports au sein d'un espace relationnel en fonction des représentations sur la manière dont cet espace doit fonctionner. Il s'agit d'abord, et notamment au niveau international, d'aboutir à un consensus entre les acteurs sur la manière de concevoir le monde, ou plus précisément sur la nature de leurs relations. On comprendra alors combien les arènes de négociation et d'objectivation comme la $\mathrm{CCO}$, qui détiennent une autorité particulière dans la mise en forme des représentations, sont des objets d'études privilégiés.

\section{La configuration de la CCO}

Il faut l'entendre comme un espace de négociations entre des individus qui mettent en jeu des intérêts différenciés et sont pris dans des relations d'interdépendances complexes. Il existe d'une part des relations d'interdépendances personnelles, propres aux individus appartenant à 
la Commission et placés dans un espace temporellement et géographiquement fixé, et d'autre part les relations d'interdépendances que chaque individu entretient avec d'autres espaces relationnels, à commencer avec les Etats qu'ils représentent. Enfin, il existe aussi un ensemble de relations d'interdépendances plus diffuses qui lient la Commission à d'autres institutions comme le secrétariat de la SDN ou le Comité central permanent du trafic de l'opium. Bien que ces dernières relations d'interdépendances se manifestent la plupart du temps au travers des individus, elles ont la particularité de s'imposer à l'ensemble des membres de la commission et de forcer en quelque sorte une unité, ou une identité spécifique ${ }^{11}$.

Les relations entre les membres de la CCO sont guidées par l'idée que chacun d'eux se fait des intérêts de l'Etat qu'il représente. Cette idée se constitue d'une part à partir de la position fermement établie qu'ils sont tenus de défendre et d'autre part quand ils sont confrontés à un problème pour lequel rien n'a été prévu, en fonction de leur perception du problème des drogues issue de leur «sensibilisation à la question » et qu'ils considèrent comme étant de l'intérêt de leur Etat. En effet, les membres de la Commission deviennent ou sont des experts reconnus sur la question des drogues, ce qui n'est pas toujours le cas des administrations nationales ${ }^{12}$. En tant qu'expert leur avis est écouté et bien souvent la position d'un Etat se fonde sur les observations faites par son représentant. En ce sens, on ne peut poser les représentants des Etats comme de simples négociateurs servant des intérêts établis par devers eux. Ils participent au plus haut niveau à l'élaboration des positions des Etats en fonction de leurs propres représentations sur la question et - ce qui est souvent lié - ce qu'ils estiment être de l'intérêt de la Nation, même si cet intérêt concerne des intérêts privés comme ceux des fabricants de stupéfiants. On se reportera à titre d'exemple aux archives du ministère des Affaires étrangères sur la manière dont est constituée la position de la France $^{13}$. La position française s'élabore la plupart du temps, à partir de la demande du représentant de la France: Gaston Bourgeois; une demande qui peut être soit une demande de renseignement ou tout simplement une demande de directive. Le service français de la SDN se charge alors de contacter les services des ministères concernés et organise éventuellement une réunion interministérielle. On perçoit très bien que l'impulsion vient du représentant de la France et que les administrations nationales sont plus au service du premier que l'inverse. On peut supposer que ce cas de figure se retrouve dans les relations de la plupart des Etats industrialisés, et ce d'autant plus quand le représentant est en même temps le chef d'un service directement concerné par le problème des drogues. C'est le cas en particulier du Dr. Carrière (Suisse) et du Dr. Kahler (Allemagne), tous deux responsables des services d'hygiène publique, en charge notamment des questions relatives à la fabrication pharmaceutique.

\section{La composition de la CCO}

Il est apparu nécessaire de présenter les membres de la Commission sous forme d'un tableau synoptique (Schéma III) afin de bénéficier d'une vision d'ensemble et de pouvoir s'y reporter librement à tout moment pour en connaître la composition à telle ou telle session.

1 Cela est particulièrement visible dans les « discussions » entre la Commission et le Conseil. Par exemple en 1922 le Conseil demande à la Commission de modifier son dernier rapport. Le Président invite l'ensemble des membres de la Commission à prendre position sur ce point. En cela il participe à la construction d'une identité abstraite des membres en tant que Commission. D'autre part le Président rappelle qu'il a au préalable essayé de négocier avec le Secrétaire de la SDN l'acceptation du rapport dans sa forme originale. Quels qu'aient été les résultats de ces pourparlers, ce qui est intéressant de noter ici, c'est le médiateur individuel qui concrétise les relations d'interdépendances entre les institutions.

12 Rappelons que c'est justement durant l'entre deux guerres que sont créées, dans les pays industrialisés, des administrations spécialisées dans le traitement du problème des drogues.

13 Archives du Ministère des affaires étrangères, SDN, Secrétariat Général, Opium, IM.1.1616-1618. 


\begin{tabular}{|c|c|c|c|}
\hline Sessions du CCO & 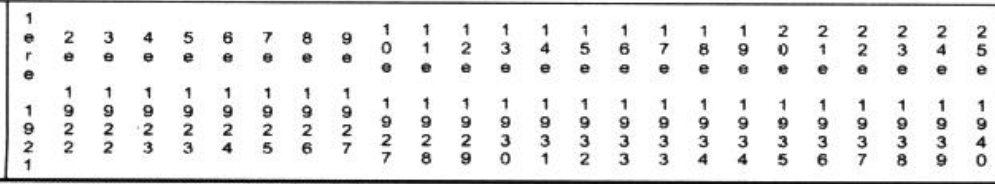 & $\begin{array}{llllllllllllllllll}1 & 2 & 5 & 1 & 2 & 3 & 4 & 5 & 6 & 7 & 8 & 9 & 1 & 1 & 1 & 1 & 1 & 1 \\
0 & 0 & 0 & 0 & 0 & 0 & 0 & 0 & 0 & 0 & 1 & 2 & 3 & 4 & 5 \\
0 & 0 & 0 & 0 & 0 & 0\end{array}$ & $\begin{array}{l}\text { morphine en } \\
\text { tonnes/opium en } \\
\text { centaine de tonnes }\end{array}$ \\
\hline \multicolumn{4}{|c|}{$\begin{array}{l}\text { Convention de La Haye 1912- } \\
\text { Convention de Geneve } 1925 \text { (28) }\end{array}$} \\
\hline \multirow{2}{*}{\multicolumn{4}{|c|}{ Convention pour la limitation 1931 (33) }} \\
\hline & & & \\
\hline $\begin{array}{l}\text { Etats } \\
\text { Chine }\end{array}$ & IS.E. M. Cha Hain Chu & & Etats \\
\hline France & Is.F. M. Bourgeols & $78,599 \mathrm{t}$ & Chine \\
\hline Grande Bretagne & Sir Matcom Detevinane & $40,358 \mathrm{t}$ & $\begin{array}{r}\text { France } \\
\text { Grande Bretagne }\end{array}$ \\
\hline Inde & Sir Jotin Campletl & $6477,248 \mathrm{t}$ & $\begin{array}{r}\text { Grande Bretagne } \\
\text { Inde }\end{array}$ \\
\hline Japon & IS.E.M. Sato I I & $38,035 \mathrm{t}$ & Japon \\
\hline Pays-Bas & M. van Wottum & 14,468 t & Pays-Bas \\
\hline Portugal & 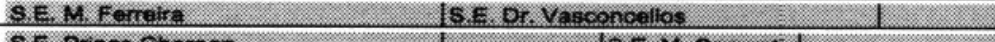 & & Portugal \\
\hline Siam/Thaillande & S.E. Prince Charoon $\quad 1$ IS.E. M. Sompat I & & Siam/Thaîlande \\
\hline Allemagne & 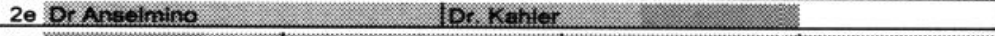 & $147,608 \mathrm{t}$ & Allemagne \\
\hline Yougoslavie & 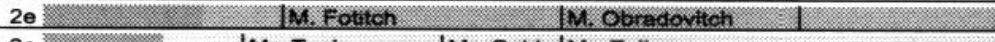 & $951,400 \mathrm{t}$ & Yougoslavie \\
\hline USA & 2e $\quad \mid$ Mr. Tuck $\quad \mid$ Mr. Caldwi Mr. Fuller & $105,013 \mathrm{t}$ & USA \\
\hline Bolivie & 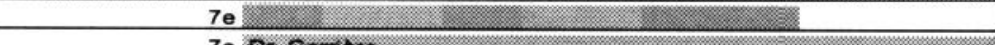 & & Bolivie \\
\hline Suisse & $7 e$ Dr. Cambere & $42,659 \mathrm{t}$ & Suisse \\
\hline Italie & 9e S.E. Sonateur Cavazzon & $3,747 \mathrm{t}$ & Italie \\
\hline Autriche & 14e Dr. Schivite & & Autriche \\
\hline Belgique & $14 \mathrm{e}$ S.E. M. Camor & & Belgique \\
\hline Egypte & 13e T. W. Russal Racha & & Egypte \\
\hline Espagne & 14e S.E. M Casares & & Espagne \\
\hline Mexique & 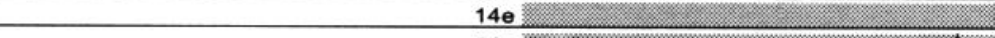 & & Mexique \\
\hline Pologne & $14 \mathrm{e}$ Dr. Chodzko & & Pologne \\
\hline Uruguay & 14e S.E. M. de Castro & & Uruguay \\
\hline Canada & $18 \mathrm{e}$ Colonel Shaman & & Canada \\
\hline Perse/Iran & 18e M. Pilossian I & $6898,023 \mathrm{t}$ & Perse/lran \\
\hline Suède & 180 & & Suède \\
\hline Turquie & $18 \mathrm{e}$ & $3344,716 \mathrm{t}$ & Turquie \\
\hline Bulgarie & $22 \theta$ & & Bulgarie \\
\hline Pérou & 228 & & Pérou \\
\hline Grèce & 230 & & Grèce \\
\hline Hongrie & 23e & & Hongrie \\
\hline Tchécoslovaquie & $23 e$ & & Tchécoslovaquie \\
\hline Assesseurs & & & \\
\hline Sir John Jordan & $=0$ & & \\
\hline Mrs Hamilton Wright (USA) & $\log ^{2}$ & & \\
\hline Brenier (France) & 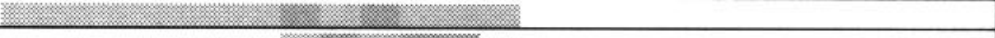 & & \\
\hline Colonel Woods (USA) & 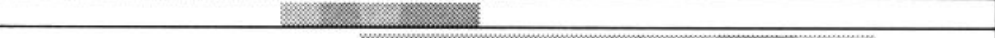 & & \\
\hline Lyall (GB) & 2 & & \\
\hline Sirks (Pays-Bas) & ( & & \\
\hline Myttenaere (Belgique) & 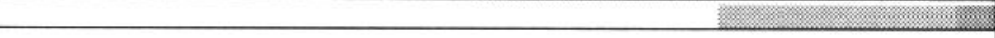 & & \\
\hline Secrétariat & & & \\
\hline Dame Rachel Crowdy (GB) & 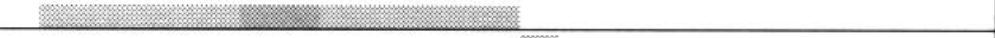 & & \\
\hline M. Duncam Hall (GB) & 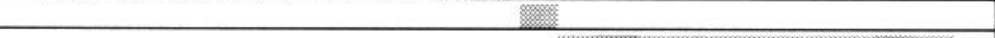 & & \\
\hline M. Ekstrand & 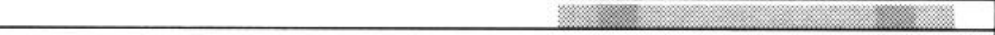 & & \\
\hline M. Renborg & $x^{2}$ & Membre non représenté & \\
\hline $\begin{array}{l}\text { Les asseurs et les représen } \\
\text { Le major Coles devient titul }\end{array}$ & $\begin{array}{l}\text { ts du secrétariat bien que membre de la Commission ne prennent pas part aux votes. } \\
\text { du siège qu'à la } 22 \text { e session }\end{array}$ & $\begin{array}{l}\text { Membre represente } \\
\text { Observateur }\end{array}$ & \\
\hline
\end{tabular}

Schéma III: Tableau synoptique des membres de la CCO. 
La qualité de membre est représentée par une bande grise qui permet de savoir quels Etats étaient présents à chacune des sessions. Les bandes en gris clair signalent que les Etats en question, bien que non membres, ont été invités à participer à la session à titre d'observateur. Les parties en gris foncé indiquent en revanche les sessions où les Etats ne se sont pas fait représenter. Enfin, les zones restées blanches désignent les périodes où les Etats concernés n'étaient ni membre, ni observateur. De plus, certains représentants ont été mentionnés en surimpression des bandes grises, soit en raison de leur longévité, soit en raison du rôle particulièrement important qu'ils ont joué (les deux sont souvent liés). Les petits traits noirs qui encadrent les noms, signalent leurs dates d'arrivée et de départ.

En vis-à-vis, est représentée l'implication des Etats dans la production ou la fabrication de stupéfiants entre 1925 et 1938. Cependant pour des raisons de lisibilitć ct de simplicité, seule la production d'opium et la fabrication de morphine ont été retenues. Ce choix se justifie par le fait que ces deux substances sont les plus significatives sur le plan économique. La production de coca et la fabrication de cocaïne sont peu importantes au regard de celles des opiacés. En outre, la morphine est la substance de base à partir de laquelle on tire l'héroïne et la codéine (les deux autres opiacés soumis à contrôle). Il suffit de connaître la fabrication de morphine d'un pays pour avoir une idée de son implication dans la fabrication totale d'opiacés. Un autre arbitrage a été fait en ce qui concerne les pays qui sont à la fois producteur d'opium et fabricant de morphine. Seule l'implication la plus significative a été retenue. Par exemple l'Inde et la Yougoslavie qui sont ou qui deviennent fabricant de morphine sur la période, sont avant tout des producteurs d'opium. C'est pourquoi nous avons négligé leur fabrication, assez faible d'ailleurs au regard des autres pays fabricants. Bien que de nombreux Etats se soient peu à peu engagés dans la fabrication de stupéfiants, cette dernière n'a pas été représentée en raison de sa faible importance. Enfin, l'URSS bien que gros fabricant et producteur n'a pas été reporté sur le schéma, du fait de sa non représentation au sein de la Commission que ce soit en tant que membre ou en tant qu'observateur.

Comme il était difficile de montrer la production ou la fabrication année par année, on a préféré l'indiquer de manière cumulée. Les chiffres fournis ne prétendent à aucune exactitude. Ils ont été établis d'après les déclarations faites par les Etats à la SDN entre 1925 et $1938^{14}$, nous nous sommes contenté de les compiler afin d'avoir une vision d'ensemble. Si nous reproduisons ces chiffres c'est qu'ils ont été utilisés par les membres de la CCO durant leurs travaux et ont même servi de base de discussions à la Conférence de 1931 sur la limitation. Ils avaient une signification pour les acteurs de l'époque, qui reconnaissaient grâce à eux une partie de l'intérêt et de l'autorité qu'avait un Etat à participer au contrôle international des drogues. C'est en ce sens qu'ils sont particulièrement intéressants. On peut tout de suite signaler, comme le montre d'ailleurs le tableau, que ceux qui dominent la CCO par leur ancienneté et leur longévité sont majoritairement les plus gros Etats fabricants.

\section{La constitution des liens d'interdépendances}

La CCO en tant qu'instance de représentation des Etats objective les liens d'interdépendances de ces derniers. Ces liens se construisent peu à peu à mesure que les problèmes sont mis en formes et vécus comme devant être absolument résolus. C'est le cas notamment du contrôle de la fabrication pharmaceutique et de son commerce international. Tous les Etats occidentaux se sont engagés au début du siècle dans la prohibition des drogues en dehors des usages médicaux. Or la fabrication et la vente en gros de ces substances sont encore en 1920 totalement libres avec comme conséquence le détournement d'importantes quantités de stupéfiants vers

14 Bien que certains chiffres existent avant et après ces dates ils ne sont pas suffisamment complets pour être utilisés. 
les usages illégitimes. Par exemple un fabricant de stupéfiants allemand ou français peut tout à fait légalement vendre des tonnes d'héroïne à un client aux Pays-Bas qui revendra ces substances à des trafiquants ${ }^{15}$. La relation d'interdépendance qui se crée alors entre les Etats fabricants réside dans l'engagement de chacun à limiter la vente d'abord puis la fabrication aux seuls usages légitimes. Il suffirait en effet d'un Etat qui ne respecte pas cette mesure pour que non seulement les autres soient menacés par l'introduction en fraude de stupéfiants, mais surtout pour que les fabricants de stupéfiants du pays fautif pratiquent une concurrence déloyale à l'égard des autres. Les différentes conventions adoptées sur les drogues manufacturées à cette époque ont donc pour but principal de formaliser et d'entériner dans le temps ces relations d'interdépendances, afin de s'assurer que chacun joue bien le «jeu ». C'est donc parce que ces relations d'interdépendances n'cngagent pas seulement les «Etats» mais l'ensemble des types d'acteurs que nous avons désignés que nous pouvons parler de configuration du contrôle international des drogues. La CCO, parce qu'elle résume ou manifeste l'ensemble de ces relations, est un objet d'analyse privilégié. On perçoit, par exemple, très clairement les liens d'interdépendances qui lient la fabrication à la consommation, les Etats fabricants entre eux d'abord, puis aux Etats consommateurs ou encore les industries aux Etats et même les industries entre elles. Il en est de même dans les relations Etat/médecins, médecins/pharmaciens et professions médicales/patients.

\section{Objectivation et structuration du contrôle international de la fabri- cation des stupéfiants}

Bien qu'il se soit construit extrêmement rapidement, l'espace du contrôle international des drogues ne s'est pas présenté d'emblée lorsque s'est posée la question des drogues au niveau international. La configuration dégagée s'est formée peu à peu à mesure que les enjeux se précisaient et s'objectivaient. Il est possible de retracer la construction du contrôle international des drogues à partir de deux objectivations statistiques qui ont été à la base de tous les travaux de la Commission consultative de l'opium: l'évaluation des besoins de la consommation légitime d'une part et celle de la fabrication des stupéfiants d'autre part. Ces deux approches corrélatives nous renseignent assez précisément sur les enjeux du contrôle international des drogues et notamment sur l'un de ses buts: la limitation de la fabrication des stupéfiants aux seuls besoins légitimes.

\section{Les enjeux de l'objectivation statistique}

En 1921, lors de la première session de la Commission, le problème des drogues est encore assez confus. On ne sait pas vraiment de ce dont on parle. Ou plutôt si, il s'agit d'éradiquer la consommation non médicale. Mais le phénomène n'est pas clairement identifié. On sait que des quantités d'opium sont produites, préparées et vendues chaque année aux opiomanes, surtout en Asie. On sait aussi que malgré l'interdiction prononcée dans la plupart des pays occidentaux de consommer des stupéfiants en dehors des prescriptions médicales, ces mêmes pays n'exercent aucun contrôle sur la fabrication de morphine, d'héroïne ou de cocaïne. On ne connaît ni la consommation légitime, ni la consommation illégitime, ni les quantités produites

is Cet exemple s'appuie sur une affaire ayant réellement existé - «l'affaire Naarden» - et qui fut présentée aux membres de la CCO lors de sa $12^{e}$ session (1929). Voir à ce propos: SDN, Commission consultative de l'Opium et autres drogues nuisibles, Rapport au conseil sur les travaux de la douzième session tenue à Genève du 17 janvier au 2 février 1929. C.33.1929. XI. 
par les industriels (qui sont alors les seuls à produire des stupéfiants manufacturés, le laboratoire clandestin n'apparaîtra que plus tard). La convention de La Haye (1912) engageait chaque Puissance signataire à transmettre ce type d'information au gouvernement néerlandais, alors chargé des fonctions de secrétariat jusqu'à leur transfert à la SDN. Les substances visées étaient l'opium, la morphine, l'héroïne et la cocaïne. La guerre aidant, aucune donnée ne fut transmise. Or, le problème des drogues tant qu'il n'est pas chiffré n'est pas mesurable. L'objectivation chiffrée ou statistique permet une manipulation intellectuelle du problème, il devient quantifiable, identifiable. Cette tendance de l'objectivation statistique, si elle est propre aux Etats ${ }^{16}$, est aussi une constante du contrôle international des drogues. Aujourd'hui encore le Programme des Nations Unies pour le contrôle international des drogues s'emploie régulièrement à essayer de quantifier les usages illicites des stupéfiants. Plus qu'un reflet fidèle de la réalité, l'intérêt principal de l'objectivation statistique réside dans les usages qui en est fait et dans les significations que lui attribuent les acteurs.

\section{L'objectivation de la consommation légitime}

Dès sa première session, la CCO recommande: Que le Conseil étudie la possibilité d'une enquête sous les auspices de l'Organisation internationale d'hygiène de la Société des Nations ou de tout autre organisation, pour déterminer approximativement la quantité moyenne de drogues mentionnées au chapitre III [opium médicinal, morphine, héroïne et cocaïne] de la Convention [de La Haye], nécessaires pour les besoins de la médecine et autres besoins légitimes ${ }^{17} .{ }^{18}$ Les membres de la Commission justifient ce projet d'évaluation par le fait que si les Etats se sont engagés à restreindre l'emploi des drogues auxquelles s'appliquent le chapitre III de la Convention [...]. Ils n'ont aucune donnée sur la nature des besoins légitimes ... ${ }^{19}$. Ce projet d'évaluation de la CCO nous indique clairement la manière dont les Etats occidentaux, et par mimétisme les institutions internationales, procèdent pour agir sur le monde. Il s'agit d'abord de mesurer le phénomène sur lequel les acteurs veulent agir. Mais cette mesure n'est pas neutre; elle ne fait pas que s'apposer à la réalité; elle construit en définitive le phénomène par une objectivation rationnelle fournie ici par les statistiques. Elle se distingue de la connaissance intuitive propre à un acteur en particulier, en s'imposant comme scientifiquement valable et à ce titre universellement vraie, donc incontestable. Toutefois, cette objectivation ne désigne pas une vérité en soi, elle attire l'attention sur un aspect particulier de la réalité qui représente un certain intérêt pour les acteurs qui promeuvent cette objectivation. La promotion de l'évaluation de la consommation légitime est surtout le fait du représentant britannique: Sir Malcom Delevingne. Ce dernier poursuit, alors, un but précis avec cette évaluation. Il s'agit d'établir scientifiquement une équation entre la quantité totale de stupéfiants produite ${ }^{20}$ chaque année et

16 Briand E., 1994.

17 Il est intéressant de remarquer ici que les usages légitimes ne se limitent pas encore pour les membres de la Commission aux seules fins médicales et scientifiques. En effet, il n'existe pas encore à cette époque de position définitive sur le traitement des toxicomanes. Les avis sont partagés. Certains considèrent qu'il est nécessaire de les inclure parmi les consommateurs légitimes, avec pour objectif de les désintoxiquer, $d$ 'autres pensent au contraire que la solution doit être la prohibition totale de tous les usages non médicaux.

18 SDN, Commission consultative du trafic de l'opium. Rapport de la commission consultative du trafic de l'opium, première session, Genève, 2-5 mai 1921. C.28.11.157.1921.XI.

19 SDN, Commission consultative du trafic de l'opium. Rapport de la commission consultative du trafic de l'opium, première session, Genève, 2-5 mai 1921. C.28.11.157.1921.XI.

20 La Commission fait envoyer dès sa première session un questionnaire à tous les Etats parties à la Convention de La Haye pour qu'ils indiquent les quantités de substances visées par la Convention qu'ils ont fabriquées, importées et exportées. 
les besoins légitimes du monde. La différence entre les deux chiffres devrait indiquer un surplus de fabrication. Or c'est ce surplus de fabrication qui est justement soupçonné d'alimenter le trafic illicite. Le principe de l'équation sera clairement posé dans le rapport de la Commission sur l'état de ses travaux durant la deuxième session:

Tant qu'il se fabriquera des stupéfiants en quantités supérieures aux besoins légitimes, il subsistera un danger sérieux, quelles que soient les mesures de contrôle que l'on puisse mettre en vigueur, de voir le surplus s'écouler par des voies illégitimes.

Par cette déclaration, la CCO construit la nature du problème et esquisse implicitement la dimension internationale de la solution. L'assertion «qucllcs quc soicnt les mesures de contrôle» vise ici surtout les modes de contrôle nationaux. La solution internationale préconisée par le représentant britannique n'est pas reproduite dans le rapport mais dans les procès verbaux de la $2^{\mathrm{e}}$ session. Pour ce dernier, il faut limiter directement la production des fabricants de stupéfiants par l'instauration d'un monopole international de la fabrication des stupéfiants dans lequel chaque Etat déjà fabricant se verrait octroyer une quote-part ${ }^{21}$. Le projet prévoyait une entente directe entre les entreprises fabriquant des stupéfiants afin qu'elles se répartissent le marché. Toute nouvelle entreprise prétendant par la suite participer au marché légal des stupéfiants aurait été contrainte de négocier sa quote-part avec les fabricants déjà sur le marché. Ce qui, pour un observateur de l'époque, ne constituait rien d'autre qu'une forme de monopole dans lequel il serait très difficile d'entrer une fois ce dernier institué ${ }^{22}$. A compter de cette date et jusqu'à la Conférence de 1931 sur la limitation, la question de la limitation de la fabrication des stupéfiants tournera autour de cette question des quote-parts et de l'instauration d'un monopole international des fabricants.

Pourquoi le représentant britannique développe-t-il cette stratégie ? Nous n'avons malheureusement que trop peu de documents pour l'expliquer de manière certaine. Cependant, nous savons que l'industrie des stupéfiants britannique est, après la première guerre mondiale, dans une phase de déclin. Ses parts de marché ne cessent de décroître ${ }^{23}$. Par exemple, la fabrication de cocaïne qui débute en 1928 dans les Etablissements May and Baker se fait sous l'impulsion de leur nouveau propriétaire: les Etablissements Poulenc Frères qui ont acquis la société en $1927^{24}$. Le fait d'instaurer un monopole international basé sur un système de quote-parts reviendrait à fixer dans le temps et de manière quasi intangible les parts de marché de chacun au moment de l'adoption du système. On comprend alors quel intérêt peut avoir une industrie en déclin pour un tel projet.

Confiée à un sous-comité mixte composé de deux membres du Comité d'hygiène publique de la $\mathrm{SDN}^{25}$ et de deux membres de la $\mathrm{CCO}^{26}$ la question des évaluations se heurte bien vite à des difficultés techniques inhérentes à la nouveauté de cette démarche. Chaque gouvernement interrogé sur cette question développe sa propre technique d'évaluation: l'Angleterre se contente d'ajouter foi aux déclarations des fabricants, considérant que leur fabrication est

21 Le projet est encore plus clairement expliqué dans le rapport du comité préparatoire des conférences de 19241925, où Sir Malcom Delevingne expose plus en détail son projet: LEAGUE OF NATIONS, Report of the Opium preparatory Commitee, Geneva, July 20 ${ }^{\text {th }}$, 1925. C.415.M.149.1925.XI.

22 Höjer, 1925, p. 213 s.

23. Sa part dans la fabrication mondiale de morphine passe de $15,4 \%$ en 1925 à $4,8 \%$ en 1938 , et de $25,8 \%$ en 1925 à $13,4 \%$ en 1938 pour les exportations. Chiffres établis d'après les déclarations du gouvernements britannique à la SDN. Notamment LEAGUE OF NATIONS, Analysis of the international Trade in Morphine Diacetylmorphine, and Cocaine for the years 1925-1930, 1930, C.587.M.228.1930.XI.

24 Cayez, 1988, p.89-90.

25 Le Dr Carrière (Suisse) et le Dr Chodzko (Pologne) tous deux futurs membres de la CCO.

26 Sir John Campbell (Inde) et le Dr Anselmino (Allemagne). 
uniquement destinée aux besoins légitimes, d'autres comme la France, sont incapables ou rechignent à donner des chiffres, d'autres encore comme la Suisse ont basé leur enquête sur les déclarations des pharmaciens et des hôpitaux et fournissent un chiffre particulièrement élevé en raison de la crainte de ces derniers de voir leur utilisation par trop limitée. En effet, aucun pays ne possède à l'époque d'institutions spécialisées dans la collecte de ce genre d'information. Bien qu'insatisfaisantes, les différentes enquêtes fournissent des chiffres à peu près équivalents entre des pays jugés comparables (USA, Grande Bretagne, Allemagne), soit environ $550 \mathrm{mg}$ de morphine par habitant et par an. Malgré de nombreuses réserves, les membres de la sous-commission finissent par s'entendre sur une consommation maximale de $600 \mathrm{mg}$ par habitant et par an. Cette entente, fortement encouragée par le représentant de l'Inde, est à mettre au compte de la stratégie britannique d'instaurer le plus vite possible une limitation contingentée de la fabrication. Dès sa session suivante, et bien que certains membres du Comité d'hygiène aient témoigné de leur inquiétude sur la validité de ce chiffre ${ }^{27}$, la CCO l'adopte et décide sur proposition britannique de ne plus renouveler le mandat de ses membres au sein du sous-comité. Cette dernière décision montre s'il en était encore besoin, que le but poursuivi était moins d'obtenir un chiffre exact de la consommation, qu' un chiffre quelque soit sa valeur scientifique pour justifier la mise en place d'une limitation auprès de pays qui comme la France ou les Pays-Bas y rechignaient.

\section{L'objectivation du commerce international et de la fabrication des stupéfiants}

L'évaluation de la consommation légitime n'aurait servi à rien, du moins dans la perspective britannique, si elle n'avait été mise en relation avec le deuxième terme de l'équation: la fabrication de stupéfiants. Là encore dès sa première session, la CCO recommande au Conseil l'envoi d'un questionnaire aux gouvernements parties à la Convention de La Haye, par lequel les Etats indiqueraient les quantités de stupéfiants fabriquées, importées et exportées. En raison du caractère non contraignant de la demande et surtout de l'absence d'un mode de collecte standardisé, ces renseignements parvinrent de manière incomplète au secrétariat de la $\mathrm{CCO}$ entre 1922 et $1924^{28}$. Toutefois, l'idée que la production dépassait très largement les besoins légitimes s'était imposée avant même que des chiffres définitifs ne soient établis. Ainsi la quatrième Assemblée prit le 27 septembre 1923 la résolution ( $n^{\circ}$ VI) d'inviter tous les Etats concernés à se réunir dans le but de conclure un accord pour limiter la fabrication des stupéfiants et contrôler leur transit. Les Etats présents à la conférence qui se réunit du 17 novembre 1924 au 19 février 1925, ne parvinrent pas à s'entendre sur un système de limitation directe de la fabrication. Le modèle anglais des quote-parts fut rejeté, certains pays fabricants comme la France, la Suisse ou les Pays-Bas préférant jouer la carte de la libre concurrence. Toutefois, un accord fut conclu en ce qui concernait le commerce international des stupéfiants: le système des certificats (voir schéma IV). Chaque Etat s'engageait à ne laisser entrer ni sortir aucun stupéfiant sans un certificat dûment visé par ses services. Ainsi, il devenait possible de suivre les flux des stupéfiants manufacturés à tous les points de la planète et notamment de savoir en quel endroit et à quel moment certaines quantités disparaissaient des circuits licites. La mise en

27 Ainsi au professeur Léon Bernard dc déclarer lors de la première session du Comité d'hygiène en 1923 : On en arrive ainsi à regretter d'avoir mis tant de bonne volonté à rechercher un chiffre qui ne peut avoir aucune valeur scientifique et qui peut avoir des conséquences dangereuses. Il serait ainsi plus convenable, pour les hygiénistes, de répondre à la Commission de l'Opium: Nous ne pouvons pas répondre à la question posée, car, scientifiquement, on ne peut établir le taux de consommation légitime. Ce n'est pas par cette voie que vous atteindrez le but proposé [à savoir la limitation de la fabrication]. Cité in Höjer, 1925.

28 Voir les premières compilations publiées par la SDN. Notamment SDN, Commission consultative du trafic de l'Opium. Exposé relatif à la fabrication de la morphine, d'autres dérivés de l'opium et de la cocaïne, accompagné de tableaux statistiques. O.C.101. 


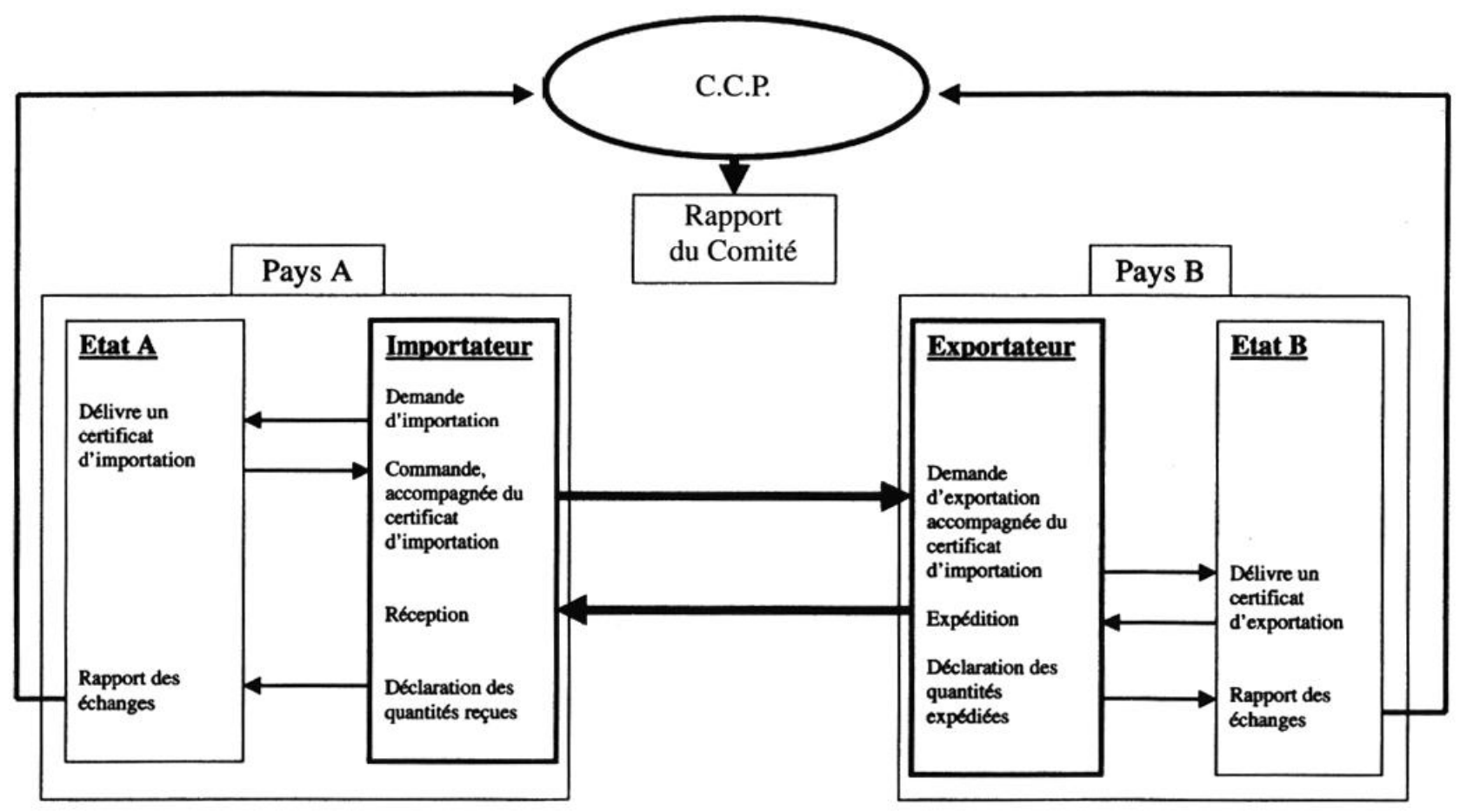

Schéma IV: Système des certificats.

place du système des certificats constitue déjà une forme de monopole commercial, puisque les entreprises déjà exportatrices à destination des marchés légitimes avaient plus de facilités pour obtenir un certificat. Un aspect essentiel du système des certificats réside dans la création du Comité central permanent de l'opium (CCP) qui entrera en fonction en 1928. Ce Comité est chargé de collecter chaque annćc les déclarations des Etats sur les quantités de stupéfiants fabriquées, importées et exportées. Il constitue l'objectivation institutionnelle de l'objectivation statistique. La création d'un organe international chargé de collecter des renseignements sur tous les Etats membres représente un moyen pour les Etats de se contrôler mutuellement. Toutefois, la CCO n'attendra pas les premiers rapports du CCP pour tenter d'avoir une idée précise du commerce international des drogues et commandera une expertise autonome sur l'état de la fabrication mondiale des stupéfiants. Lors de sa onzième session en 1928, elle reconnaît dans son rapport que les statistiques communiquées par les gouvernements ne lui permettent pas de se faire une idée exacte de la situation des drogues:

En admettant même que tous ces chiffres soient approximatifs, il semble que nous soyons encore très imparfaitement renseignés sur la fabrication de la morphine et sur les sources où $s^{\prime}$ alimente le trafic illicite dont cette drogue est l'objet ${ }^{29}$.

Ce constat est à mettre en relation avec les négociations préliminaires en vue d'instaurer le modèle anglais des quotas qui gagne avec le temps de plus en plus de partisans parmi les Etats fabricants, mais qui suscite aussi des inquiétudes se manifestant par la proposition de projets de limitation concurrents. Ainsi, lors de la même session, le représentant des Pays-Bas soumet à la Commission un projet américain de limitation de la fabrication qui rejette le principe des quote-parts. En fait, le projet en question - appelé «Plan Crane» en référence au citoyen

29 SDN, COMMISSION CONSULTATIVE DU TRAFIC DE L'OPIUM ET AUTRES DROGUES NUISIBLES, Rapport au Conseil sur les travaux de la onzième session, Tenue à Genève, du 12 au 26 avril 1928, p. 8. 
américain qui l'a présenté au gouvernement des USA - a été rédigé par M. Blanco ${ }^{30}$ ancien membre de la section du trafic de l'opium du Secrétariat de la SDN.

En septembre 1929, l'Assemblée invita la Commission à présenter un projet de limitation en vue d'une conférence à venir entre pays fabricants et consommateurs. L'enjeu qui se précise alors consiste moins à limiter la fabrication des stupéfiants aux seuls besoins médicaux et scientifique, ce qui, à cette époque, est un principe acquis par tous, qu'à déterminer qui participera à la fabrication de la quantité de stupéfiants ainsi délimitée. En d'autres termes, il s'agit d'établir comment et avec qui va s'organiser l'économie mondiale des stupéfiants. Deux projets vont alors s'affronter. Si tout le monde reconnaît que chaque Etat peut fabriquer des stupéfiants pour subvenir à la demande intérieure légitime, l'opposition se focalise autour de la question de la fabrication des stupéfiants destinés aux pays qui ne fabriquent pas. Le premier projet, dit des quote-parts, préconise l'instauration d'un monopole international des principaux fabricants qui se répartiront le marché des pays dit consommateurs. Ce projet aurait pour effet d'entériner un état de fait et de laisser aux fabricants déjà présents le soin de se répartir le marché, sans garantie pour de futurs prétendants d'obtenir une quote-part. Le second, dit plan Crane, prévoit au contraire une libre concurrence contrôlée entre les fabricants. Ces derniers pourront fabriquer autant de stupéfiants qu'il leur sera commandé pour des fins médicales et scientifiques. Le système des certificats permettant de surveiller la légitimité des commandes. Ceci dit les deux projets tendent vers la création d'une configuration oligopolistique du marché mondial des stupéfiants manufacturés. Que les fabricants participant au commerce international soient désignés formellement, comme le préconise le système des quote-parts, ou qu'ils se désignent eux-mêmes par le fait de la compétition économique, il n'en demeure pas moins que l'on reste dans une configuration fermée. Les acteurs économiques sont, en effet, limités d'une part par un plafond indépassable qui est la consommation mondiale de stupéfiants à des fins médicales et scientifiques et d'autre part par les autorisations de fabrication et d'exportation délivrées par les Etats. Ainsi, on peut considérer que les deux projets de limitation tendent vers la constitution d'une économie dirigée ${ }^{31}$ des stupéfiants qui serait dominée et organisée mondialement par les fabricants et les Etats.

Afin de répondre aux vœux de l'Assemblée, mais aussi au plan Crane, les tenants du projet des quote-parts entreprennent d'appuyer leurs propositions sur une connaissance solide de la situation des stupéfiants manufacturés dans le monde en commandant une enquête. Le but de cette étude était de démontrer que la libre concurrence entre les fabricants était à l'origine de la surproduction de stupéfiants manufacturés. Dans cette perspective, une limitation simple de la fabrication aurait été inutile si elle ne s'accompagnait pas d'une répartition préalable du marché. Une note interne du Secrétariat ${ }^{32}$ datée du 10 octobre 1930 mentionne une discussion

30 M. Blanco, de nationalité espagnole, est ce qu'on peut appeler un entrepreneur de morale. Alors qu'il était fonctionnaire de la section du trafic de l'opium, il avait pris plusieurs fois position dans des notes internes contre le projet britannique des quote-parts, arguant que ce projet ne garantissait pas les droits des pays consommateurs de s'approvisionner là où ils le désiraient et surtout qu'il ne prenait pas en compte la limitation de la production des matières premières (opium et coca). Cette lacune, considérait-il, permettrait au trafic illicite de continuer à se développer, car il serait toujours possible pour les trafiquants de trouver de la matière première à transformer. En 1929, il dirige le «Bureau anti-opium » basé à Genève. Nous avons peu de renseignement sur ce bureau et ses membres, il possédait néanmoins ses entrées auprès des instances internationales comme en témoigne ce projet parvenu jusqu'aux membres de la CCO. Archives de la SDN, 12/3196/4003.

31 Là encore, cette représentation est partagée par les acteurs de l'époque puisque l'Assemblée générale, elle-même, qualifia, en 1934, l'application des instruments de contrôle international de véritable économie dirigée des stupéfiants. Rapporté dans l'introduction de SDN, SECTION DU TRAFC DE L'OPIUM dU SECRÉTARIAT DE LA SOCIÉTÉ DES NATIONS, 1937.

32 Archives de la SDN, 12/15141/4003. 
informelle entre Sir Malcom Delevingne (le représentant britannique à la CCO), Mr. May (membre du CCP et de nationalité américaine), Mr Lyall (assesseur britannique auprès de la $\mathrm{CCO}$ ) et $\mathrm{Mr}$ Hall (auteur de la note et membre de nationalité australienne du Secrétariat). Cette discussion qui avait pour objet de produire l'étude susmentionnée (à savoir la constitution d'un tableau général de l'économie des stupéfiants manufacturés) aboutit à la désignation du docteur Anselmino (représentant allemand de la CCO jusqu'en 1928) pour mener à bien cette tâche. Une autre note ${ }^{33}$ du même auteur et datée du lendemain nous apprend que le docteur allemand accepte cette mission. Mais surtout elle fait référence à un ensemble de discussions qui permettent de repérer ceux qui, à cette date, étaient en faveur d'un monopole international des stupéfiants manufacturés:

He [Dr. Anselmino] referred back to his previous scheme for a cartel. He informed me that he had talked with Sir Malcom Delevingne, Mr. van Wettum and others, and all had agreed that it was essential to secure a general agreement amongst the manufacturers with regard to quotas and distribution of their products throughout the world.

Ces notes permettent de mettre en lumière plusieurs choses. Tout d'abord, on peut remarquer que le cercle des tenants du système des quote-parts s'est élargi, par rapport à 1925, puisqu'on peut au moins citer les Pays-Bas et l'Allemagne ainsi que d'autres («others») qui sont vraisemblablement la France et la Suisse d'après leur position lors de la Conférence de Londres en $1930^{34}$. De plus, il faut noter l'alliance qui se noue entre les membres des institutions suivantes: la Commission, le Comité central permanent et le Secrétariat. Au regard de l'identité des personnes citées, on ne peut que rappeler la force des liens nationaux: deux des interlocuteurs mentionnés sont britanniques. On pourrait même en citer un quatrième: Dame Rachel Crowdy, Chef de la section du trafic de l'opium et des questions sociales, à qui ces notes sont vraisemblablement adressées ${ }^{35}$. Ces documents montrent en outre la place centrale occupée par Sir Malcom Delevingne sur cette question. Quelles que soient les actions cnvisagées sa référence apparaît comme une forme de validation pour les acteurs qui le mentionnent. Enfin, ces notes nous permettent de comprendre dans quelle perspective a été faite l'objectivation statistique de l'économie des drogues manufacturées. Il s'agissait de l'utiliser pour soutenir l'idée d'une limitation passant par un système de contingentement. Le travail du Dr Anselmino servit de base à une autre étude statistique du secrétariat publiée en $1930^{36}$. Cette analyse est la première mise en forme officielle de l'économie mondiale des stupéfiants manufacturés. Elle fut utilisée, lors de la Conférence de 1931 sur la limitation, comme document de travail. L'objectivation statistique qui est publiée en 1930 a été construite et utilisée dans le but de légitimer le projet d'entente que certains pays étaient en train de mettre en place. L'idée de la mise en place d'un monopole international des drogues avait déjà été entérinée par le Conseil en mai 1930. Ce dernier, approuvant une suggestion de la Commission, engageait les pays fabricants à se concerter en vue de se répartir entre eux des quote-parts sur les stupéfiants ${ }^{37}$.

$33 \quad$ Archives de la SDN, 12/15141/4003.

34 La Conférence tenue à Londres en 1930 avait pour but d'aboutir à une entente préalable sur la répartition du marché mundial des stupéfiants manufacturés entre les pays fabricants.

35 Pour avoir remplacé Dame Rachel Crowdy comme représentant du Secrétariat lors de la $14^{\mathrm{c}}$ session, nous supposons que Mr Hall était son subordonné direct.

36 League of Nations, Analysis of the international Trade in Morphine, Diacetylmorphine, and Cocaine for the Years 1925-1930.

37 SDN, Trafic de l'opium et autres drogues nuisibles, Rapport de la réunion préliminaire des représentants officiels des pays manufacturiers, Tenue à Londres en octobre - novembre 1930, Genève, 1930, C.669.M.278.1930.XI., p. 1 et 2. 
La manière de voir et de construire le problème des drogues est inséparable des enjeux proprement économiques que recouvrent la consommation légitime des stupéfiants. Elle participe non seulement à la désignation des enjeux, mais aussi, et du même coup, à celle des acteurs autorisés à prendre part à la configuration et à la position relative de chacun.

\section{Désignation des «acteurs»}

\section{1. «Etats fabricants», «Etats consommateurs»: une typologie stratégique}

Dès les premiers temps, il fut établi une distinction de fait entre les Etats fabricants et les Etats consommateurs. Cependant, grâce à l'analyse de 1930, et à mesure que se précise la question de la limitation, apparaît une distinction de statut entre les Etats fabricants. Cette nouvelle identification des Etats entre eux constitue une nouvelle objectivation permettant de les positionner plus précisément dans l'espace du CID. Le rapport ${ }^{38}$ de la Conférence tenue à Londres en 1930 entre les Etats fabricants en vue de se répartir le marché international des stupéfiants nous apprend comment les Etats fabricants, en se représentant le monde des drogues manufacturées, ont participé à sa construction. Nous reproduisons la typologie telle que présentée dans le rapport ${ }^{39}$ :

1. «Les pays qui fabriquent pour leurs propres besoins et aussi, dans une mesure considérable, en vue de l'exportation: Allemagne, France, Pays-Bas, Royaume-Uni, Suisse. [Nous les appellerons désormais les Grands pays exportateurs] ${ }^{40}$

2. Les pays qui fabriquent pour leurs propres besoins mais qui à l'heure actuelle ne font pas d'exportation dans une mesure considérable: Etats-Unis d'Amérique, Japon, Union des Républiques soviétistes.

\section{Les pays qui ne fabriquent que pour une faible partie de leurs propres besoins: Italie.»}

Cette typologie est déterminante, car elle désigne, dans la perspective du projet des quoteparts, les Etats qui sont, a priori, autorisés à se répartir le marché international des drogues. La distinction intellectuelle opérée entre ceux qui exportent «dans une mesure considérable » et ceux qui «à l'heure actuelle ne font pas d'exportation dans une mesure considérable» revient à désigner $a$ priori les cinq pays pressentis pour se répartir le marché des exportations. Les autres Etats fabricants se voient octroyer leur seul marché intérieur. Les chiffres étant incontestables car fournis par les Etats eux mêmes et avalisés par la SDN, il devenait difficile pour un nouvel Etat de prétendre entrer sur le marché des exportations (et donc d'augmenter sa fabrication) alors même qu'il avait été admis et démontré statistiquement ${ }^{41}$ que la production mondiale dépassait largement les besoins légitimes. C'est cette logique du moins que les Grands Etats exportateurs défendirent pour justifier leur entente et tenter de la faire accepter. L'objectivation statistique est instrumentalisée ici dans le but de fixer et légitimer un rapport de force.

38 SDN, Assemblée préliminaire des pays manufacturiers des drogues, 1930, cote 12/23680/4003.

39 Le rapport mentionne, en outre, deux cas particuliers : la Turquie d'une part qui au vu de la formation de ce monopole international s'est déclarée au dernier moment comme un Etat fabricant et exportateur et l'Inde qui a exporté par le passé de grosses quantités de morphine brute à destination de l'Angleterre.

40 D'après l'analyse publiée par le Secrétariat ces pays concentreraient à eux seuls pour 1929: 97,3\% des exportations de morphine, $92,2 \%$ pour l'héroïne et $98,8 \%$ pour la cocaïne.

41 Voir le rapport susmentionné du secrétariat sur l'économie mondiale des stupéfiants: LEAGUE OF NATIONS, Analysis of the international Trade in Morphine, Diacetylmorphine, and Cocaine for the Years 1925-1930. 
Notons qu'une alliance stratégique entre des Etats comme la Grande-Bretagne, la France et l'Allemagne, qui dominaient encore avant la guerre les relations internationales dans leur ensemble, avait toutes les chances d'imposer ses vues. Et c'est bien leur manière de voir les choses qui est retenue dans ce rapport. Leur typologie est une manière de structurer la place de chacun dans l'espace des drogues manufacturées. Elle établit une hiérarchie non dite entre les différents statuts.

L'enjeu était d'autant plus crucial que le rapport de la Conférence de Londres, d'où est tirée cette typologie, devait servir de base de travail pour la Conférence internationale pour la limitation prévue en juin 1931.

\section{Les Etats membres de la commission (Schéma III)}

La désignation des Etats membres de la Commission correspond à une logique semblable, du moins jusqu'en 1931. Elle s'organise en fonction des rapports de force entre les Etats et les enjeux qui ont été identifiés comme tâche de la Commission. En reprenant le Schéma III des membres de la Commission jusqu'en 1931, nous tenterons d'expliquer la présence de chacun d'entre eux.

\section{$I^{\text {re }}$ session: Chine, France, Royaume-Uni, Inde, Japon, Pays-Bas, Portugal, Siam}

Les huit Etats membres de la Commission à ses débuts ont, semble-t-il, été désignés en fonction de leurs liens avec les problèmes de l'opium fumé en Asie. La Chine et l'Inde en tant que principaux pays producteurs et consommateurs d'opium non médicinal. Le Royaume-Uni, la France, les Pays-Bas, le Siam et le Portugal pour les régies d'opium que ces Puissances entretenaient dans leurs possessions. Le Japon enfin à cause des territoires qu'il occupe en Chine.

\section{$2^{e}$ session: Allemagne, USA et Yougoslavie}

La désignation par l'Assemblée de 1921 de l'Allemagne, des USA et de la Yougoslavie correspond à l'orientation des travaux de la $\mathrm{CCO}$ vers la question des produits manufacturés. L'Allemagne et les Etats-Unis sont en effet les deux premiers fabricants mondiaux de stupéfiants et la Yougoslavie l'un des principaux producteurs d'opium destiné aux fabricants de stupéfiants. Les USA, en raison de leur politique isolationniste, ne siégèrent qu'à titre d'observateur.

\section{$7^{e}$ session. Bolivie et Suisse}

Bien que la Bolivie ne se fît pas représenter avant la $9^{\mathbf{e}}$ session, la désignation de ces deux nouveaux Etats est à mettre au compte de la formation de l'espace spécifique du contrôle de la fabrication. La Suisse est l'un des principaux pays fabricants avec 42 tonnes de morphine entre 1925 et 1938 et la Bolivie est invitée en tant que producteur de coca à destination des pays manufacturiers.

\section{$9^{e}$ session: Italie}

La présence de l'Italie (dernier arrivé des Etats fabricants) au sein de la CCO clôture du moins temporairement la configuration formée par les Etats fabricants. La typologie des Etats fabricants telle qu'elle est produite dans le rapport de la Conférence de Londres revient en fait à classer entre eux les Etats fabricants membres de la CCO dès 1927. Cette typologie peut donc être considérée comme l'objectivation formelle de la configuration des Etats fabricants. Un rapide coup d'œil sur le Schéma III nous permet d'avancer que cette configuration des Etats fabricants domine très nettement la $\mathrm{CCO}$ (8 Etats fabricants, si on ne compte pas l'Inde, contre 6 pays non fabricants). 


\section{$14^{e}$ session: Autriche, Belgique, Egypte, Espagne, Mexique, Pologne, Uruguay}

Ces sept pays ont été choisis en fonction de leur statut d'Etat non fabricant conformément à une résolution de la dixième Assemblée (24.09.29). Désignés lors de la onzième Assemblée, ils correspondent assez exactement à ceux que la Commission avait mentionnés à titre indicatif dans son rapport sur les travaux lors de sa treizième session (1930). Une note du représentant français adressée au Service français de la Société des Nations à Paris nous apprend que le choix des Etats d'Amérique latine a été fait en fonction du soutien qu'ils pourraient apporter au projet des quote-parts. Toutefois, on ne peut dire que leur désignation soit uniquement une cooptation des Grands pays exportateurs. Dès leur première session, les représentants de la Belgique et de l'Espagne réagirent vivement contre le monopole qui était en train de se mettre en place.

La logique de désignation des membres peut donc se résumer de la manière suivante:

$1921-1928$

Cette période est dominée par la désignation des Etats fabricants et par la constitution d'un noyau d'Etats fabricants et exportateurs qui mettent eux-mêmes en place les projets de contrôle de la fabrication et des flux.

\section{8-1931}

Le contexte est celui de la Conférence de 1931 sur la limitation avec comme enjeu principal l'instauration ou non d'un monopole international des stupéfiants manufacturés. Certains pays dits consommateurs sont invités à se faire représenter dans le but d'avaliser l'entente entre les fabricants.

\section{$1931-1940$}

Suite à l'échec du système des quote-parts et à la crise économique internationale du début des années 1930 , de nombreux pays se mettant à produire pour leur consommation intérieure, certains d'entre eux sont alors invités à devenir membre de la Commission. Des Etats importants comme l'Allemagne, le Japon ou l'Italie quittent progressivement la $\mathrm{CCO}$ du fait de facteurs politiques externes au CID. Ils sont remplacés par des pays de moindre envergure. Certains pays producteurs d'opium ou de coca qui jusque là avaient été tenus à l'écart apparaissent, à mesure que prend forme le projet français sur la limitation de la production des matières premières (Perse, Turquie et Pérou notamment).

\section{La reconnaissance des fabricants de stupéfiants}

Un dernier type d'acteur est peu à peu reconnu à mesure que se précise l'enjeu de la limitation de la fabrication: les fabricants de stupéfiants ${ }^{42}$. Bien vite ces derniers seront autorisés, même si c'est de manière médiatisée, à intervenir au plus haut niveau dans l'élaboration des conventions internationales. Ils seront même considérés comme quasiment partie prenante au projet de limitation des Grands pays exportateurs.

La première reconnaissance institutionnelle des fabricants date de la Convention de $\mathrm{La}$ Haye. L'article 10 engage les Etats à surveiller leurs activités. La Convention de 1925, va plus loin et impose aux Etats d'enregistrer et de déclarer les établissements autorisés à fabriquer les stupéfiants. Ces modes de reconnaissance qui s'exercent par le contrôle des Etats sur les activités des entreprises, ne doivent pas cependant dissimuler un autre type de reconnaissance qui est celui de la garantie de leurs intérêts économiques.

42 Il faudrait faire une mention spéciale sur le personnel des organes internationaux qui prennent de plus en plus d'importance à mesure que s'accentue la dépendance des Etats vis-à-vis de leur travail de secrétariat et que leur est reconnu une véritable expertise sur le problème des drogues. 
Dès sa sixième session la $\mathrm{CCO}$ admet le principe suggéré par le représentant de l'Allemagne et soutenu par le représentant britannique que les fabricants des divers pays devraient engager des négociations privées, afin d'arriver à un accord amiable sur la question de la base d'évaluation du prorata des quantités de drogues à fabriquer ${ }^{43}$.

Il apparaît clairement que l'espace du contrôle international des drogues ne serait pas qu'une affaire d'Etat; les intérêts privés y auraient leur place. Cette place sera surtout octroyée de manière officieuse. On peut repérer dans ce processus de reconnaissance des fabricants de stupéfiants plusieurs étapes significatives. Nous en retiendrons une qui est particulièrement parlante: la reconnaissance, par certains membres de la CCO et par le Secrétariat, des cartels internationaux des stupéfiants dans l'élaboration du projet des quote-parts.

La reconnaissance des cartels des drogues est à replacer dans le contexte de la préparation de la Conférence de 1931 pour la limitation. Les notes internes, citées plus haut, rappellent que certains représentants des Etats étaient en faveur d'un cartel des fabricants. Dans une correspondance entre Dame Rachel Crowdy et certains membres de la CCO, on trouve cette reconnaissance de manière plus explicite ${ }^{44}$. En 1929, elle écrit aux représentants allemand, suisse et néerlandais de la Commission (c'est à dire le Dr. Kahler, le Dr Carrière et Mr van Wettum). Elle leur demande s'ils peuvent lui communiquer des renseignements sur un certain cartel de la cocaïne, sous prétexte que le mode de fonctionnement de ce cartel pourrait servir aux travaux de la CCO dans l'élaboration de son projet de limitation. Après avoir essuyé un premier refus sous des raisons diverses (seul Mr van Wettum avoue qu'il ne transmettra pas les renseignements demandés en raison de l'opposition des industriels néerlandais), elle obtient finalement de ses trois interlocuteurs des informations assez complètes sur les membres et le fonctionnement de ce cartel. On apprend ainsi le nom des entreprises qui participent à cette entente. Il apparaît aussitôt qu'elles sont toutes ressortissantes des cinq Grands Etats exportateurs désignés dans le rapport de la Conférence de Londres. En outre, le cartel rassemble quasiment toutes les entreprises des ces cinq Grands pays exportateurs, à quelques exceptions près. Ces documents nous montrent que l'entente qui est en train de se forger entre certains Etats s'appuie sur celle des industriels. Les deux types d'acteurs sont intimement liés et fonctionnent sur le mode des relations d'interdépendances. Les industriels sont dépendants des Etats pour la reconnaissance officielle de leur entente, et les Etats sont dépendants des industriels pour qu'ils s'engagent à ne plus alimenter le trafic illicite, mais aussi en fonction des liens divers que le personnel de l'Etat entretient avec la grande industrie. Enfin du point de vue de la reconnaissance, il faut noter que la nomination des entreprises, loin de constituer une dénonciation, est d'abord une élection.

Le fait acquis que les Grands pays exportateurs désirent s'appuyer sur une entente entre les industries tend à nous faire penser que seules les entreprises appartenant à ce cartel seront dorénavant autorisées à exporter des stupéfiants. C'est pourquoi on peut considérer que cet acte de nomination est aussi de la part des Etats et du Secrétariat un acte de désignation et de reconnaissance. Une autre correspondance au printemps $1930^{45}$ révèle qu'un cartel des opiacés est lui aussi sur le point de voir le jour. Les négociations entre les industriels seront cette fois-ci officiellement reconnues par le Conseil lors de sa session de mai 1930. En suivant l'avis de la $\mathrm{CCO}$, le Conseil demandait que les gouvernements des pays fabricants ou les fabricants eux$\underline{\text { mêmes }}^{46}$ examinent à l'avance la répartition des contingents [des pays fabricants] et envisa-

\footnotetext{
43. SDN, Commission consultative du trafic de l'Opium, Rapport au Conseil sur les travaux de la sixième Session de la Commission, 4-14 août 1924.

44 Archives de la SDN, 12/15375/15375.

45 Archives de la SDN, 12/15375/15375.

46 Souligné par nous.
} 
gent les arrangements nécessaires pour assurer une répartition convenable entre les pays consommateurs des stupéfiants fabriqués. De fait, les industriels continuèrent leurs négociations durant tout l'été 1930. Réunis à Londres, au même moment que les représentants des Etats, leur répartition du marché servit de base de travail à la Conférence des Etats. La Conférence de Londres est sans doute le moment où se font le plus sentir les interdépendances, sous le mode d'une alliance stratégique, entre les fabricants et les Etats. Un représentant des industriels (siégeant en tant que tel) assista même à plusieurs séances de la Conférence des Etats.

Ces interdépendances entre ces deux types d'acteurs n'étaient cependant pas dépourvues d'ambivalence. L'ambiguïté qui préside à la reconnaissance des fabricants de stupéfiants par les institutions internationales, donc en partie par les Etats, est difficile à reconstruire dans son cnsemble par manque de documents. Cependant, on peut avancer deux explications complémentaires. La première tient dans le fait que les fabricants sont régulièrement accusés par la presse de participer ou d'alimenter le trafic illicite: chose dont la CCO se fait régulièrement l'écho ${ }^{47}$. Les Etats ne peuvent donc s'afficher comme étant particulièrement liés avec les fabricants. D'un autre côté, ces derniers représentent d'importants intérêts économiques que tous les Etats cherchent à préserver. Cette ambivalence peut se résumer par cette déclaration du représentant indien, Sir John Campbell, lors de la Conférence de Londres. La question est alors de savoir si dans le système des quote-parts, le bureau international qui sera créé pour répartir chaque année la fabrication de stupéfiants devra être confié aux fabricants ou non. Sir John Campbell déclare:

L'opinion publique dira certainement, pour le dire en quelques mots, en faisant cela nous employons les cambrioleurs pour garder la maison. [...] D'ailleurs, le système d'employer les cambrioleurs pour garder la maison, n'est peut-être pas un mauvais système, il est couramment appliqué aux Indes [...]. Mais il faut que nous nous rendions compte de cette difficulté que nous aurons avec l'opinion publique lorsqu'elle verra cette proposition concernant le bureau, et pour moi, je considère que dans la forme définitive de ce projet, il faudra prévoir une espèce de liaison, une espèce de contrôle gouvernemental à exercer sur ce bureau. (Traduction SDN) ${ }^{48}$

La deuxième explication, en germe dans la déclaration du représentant indien, est que les Etats ne tiennent pas à se départir de leur rôle central sur la question des stupéfiants. Il ne s'agit pas là d'une volonté abstraite de l'entité étatique, mais de la variété des intérêts qui se manifestent sur la question au sein de l'Etat et des nombreux domaines où il intervient au nom de ces mêmes intérêts. La Suisse nous offre une illustration particulièrement frappante de cette ambivalence, manifestée par l'attitude de ses hommes politiques. Lors d'une séance du parlement fédéral, le $1^{\text {er }}$ décembre 1924, le Conseiller Motta est vivement interpellé par le député Micheli sur les liens que la délégation suisse entretient avec les industriels. Nous sommes alors au début de la deuxième Conférence de l'opium tenue à Genève et qui débouchera sur la Convention de 1925, celle-là même qui mis en place le système des certificats. La réponse du Conseiller Motta est révélatrice de cette position peu confortable des gouvernants sur la question des drogues à cette époque:

Je suis persuadé qu'entre la lutte nécessaire contre le danger de l'opium et le respect des intérêts des industriels il n'y a pas d'antinomie. Vous pouvez faire confiance au Conseil fédéral. Il ne négligera en rien la tâche qui est la sienne: harmoniser les intérêts moraux et les intérêts matériels ${ }^{49}$.

47 Voir les rapports de la CCO sur la période notamment ceux sur la $12^{e}$ et $14^{e}$ session.

48 Procès verbal de la Conférence de Londres, Quatrième séance, octobre 1930, archives de la SDN sous la cote 12/23680/4003.

49 Interpellation Micheli, Instructions de la délégation suisse à la Conférence internationale de l'opium, Archives de la SDN, 12A/42218/42218, 05/1917. 
La question de la limitation est liée à d'autres enjeux, comme celui de la consommation où d'autres types d'acteurs sont autorisés à prendre la parole, comme les médecins ou les entrepreneurs de morale pour ce cas précis. Les représentants des Etats manifestent ces complexités relationnelles constitutives de l'Etat moderne occidental. Il est difficile pour eux, voir impossible de prendre clairement parti pour un camp aux dépends des autres. La défense des intérêts nationaux passe rarement par l'abandon d'autres intérêts si ces derniers restent forts au plan national. Toutefois, au sein du troisième étage, l'Etat bénéficie d'une place centrale et à ce titre dominante. Cette position centrale se perçoit notamment dans le rôle d'intermédiaire qu'il assure entre les fabricants et les organismes internationaux et plus précisément dans l'accès aux processus de décision qu'il octroie ou non aux industriels. Cette position centrale est nettement perceptible au moment de la Conférence de Londres. Si les fabricants ont été sollicités pour s'entendre entre eux sur la répartition du marché, cette répartition doit se faire suivant les représentations étatiques de l'espace. Lorsque les industriels proposent une première répartition celle-ci est établie en fonction des parts de marché de chacun, c'est-à-dire en fonction d'une vision économique de l'espace et non en fonction des frontières politiques. Les Etats imposèrent alors aux industriels une répartition conforme à l'exercice de leur souveraineté.

***

Malgré la position dominante occupée par les Grands pays exportateurs au sein des organes internationaux, le projet des quote-parts fut finalement abandonné lors de la Conférence de 1931 pour la limitation. La Convention adoptée le 13 juillet 1931 reprenait en fait les principes énoncés par le plan Crane, à savoir que les exportations de stupéfiants ne seraient pas contingentées mais limitées par les commandes reçues en vue de la satisfaction des besoins médicaux et scientifiques. Il n'en demeure pas moins que la Convention de 1931 instaure une véritable limitation de la fabrication mondiale des stupéfiants dont les principes de fonctionnement sont encore à l'œuvre aujourd'hui. L'échec des Grands pays exportateurs est cependant à relativiser. Si ces derniers ne sont pas parvenus à faire entériner par une convention internationale l'accord entre leurs fabricants, ces derniers ont néanmoins maintenu leur entente jusqu'à la veille de la seconde guerre mondiale et gardé une position dominante ${ }^{50}$.

Toutefois, la Convention de 1931 doit d'abord être considérée comme l'objectivation formelle de la configuration du contrôle international des drogues. Elle fixe non seulement les usages légitimes des stupéfiants en ce qui concerne la fabrication, mais surtout les acteurs dépositaires de ces usages (les fabricants), et ceux chargés de les contrôler et éventuellement de les modifier (Etats et organismes internationaux).

Cette objectivation de la configuration ne s'est pas faite seulement durant la conférence, loin s'en faut, mais est le fruit d'un processus assez rapide dominé par un nombre réduit d'acteurs appartenant à des espaces spécifiques (Etats, fabricants de stupéfiants, organismes internationaux). C'est en fonction des enjeux dégagés et objectivés - limitation de la disponibilité des stupéfiants manufacturés aux seuls besoins médicaux et scientifiques - que s'est constitué le dernier étage de la configuration du contrôle international des drogues. Il y a donc un lien structurel entre la formation de la configuration, comprise comme un espace de relations d'interdépendances entre un nombre déterminé d'acteurs ou de types d'acteurs, et les représentations des enjeux qui organisent cette configuration et désignent les acteurs autorisés à y prendre part.

so Les Etats où sont implantés les membres des cartels des opiacés et de la cocaïne totalisent en 1938:57,4\% des exportations de morphine, $71,2 \%$ pour l'héroïne, $77,9 \%$ pour la codéine et $80 \%$ pour la cocaïne. Chiffres établis par nous d'après: SDN, Comité Central PERMANEnt DE L'OPIUM, Production et distribution des stupéfiants et de leurs matières premières avant la guerre, Genève, octobre 1944, C.24.M.24.1944.XI. 
Dans le cas particulier du contrôle de la fabrication des stupéfiants, la configuration qui voit le jour en 1933 prend la forme d'une économie dirigée des stupéfiants au niveau mondial. Cette direction de la fabrication vers la stricte satisfaction des besoins médicaux et scientifiques, aux mains des Etats, des fabricants et des organismes intergouvernementaux, fonctionne, au moins jusqu'à la veille de la seconde guerre mondiale, de manière oligopolistique.

François-Xavier Dudouet Laboratoire d'analyse des systèmes politiques (CNRS)

Université Paris X Nanterre 200, avenue de la République 92001 Nanterre Cedex

\section{BIBLIOGRAPHIE}

BACHMANN C., COPPEL A., Le Dragon domestique: Deux siècles de relations étranges entre l'Occident et la drogue, Paris, Albin Michel, 1989.

BECKER H. S., Outsiders : Etudes de sociologie de la déviance, Paris, Métailié, 1985 (1963).

BENSUSSAN I. J., L'opium : considérations générales histoire-géographie-chimie fabrication et usage de l'opium et études économiques, sociales et législatives, Paris, Vigot Frères, 1946.

BERRIDGE V., GRIFFITH E., Opium and the People: Opiate Use in Nineteenth-Century England, New Haven, London, Yale University Press, 1987.

BOURDIEU P., Les modes de domination, Actes de la Recherche en sciences sociales, 2-3 juin 1976.

BOUSSEL P., History of pharmacy and pharmaceutical industry, Paris, Lausanne, Asklepios Press, 1983.

BRIAND E., La mesure de l'Etat, Paris, Albin Michel, 1994.

BRUUN K., PAN L., REXED I., The Gentlemen's Club, International Control of Drug and Alcohol, Chicago, London, The University of Chicago Press, 1975.

CAYEZ P., Rhône-Poulenc 1895-1875: Contribution à l'étude d'un groupe industriel, Paris, Armand Colin, Masson, 1988.

Convention internationale de l'opium signée à La Haye le 23 janvier 1912.

Convention de l'opium signée à Genève le 11 février 1925.

Convention pour la limitation de la fabrication des stupéfiants signée à Genève le 13 juillet 1931 .

ELIAS N., Qu'est-ce que la sociologie?, Paris, éd. de l'Aube, 1991.

HÖJER O., Le trafic de l'opium et autres stupéfiants : Etude de droit international et d'histoire diplomatique, Reims, Paris, Spes, 1925.

LACROIX B., Objectivisme, objectivation et ordre social, in Traité de Science Politique, GRAWITZ M., LECA J., Eds, Tome 1, Paris, PUF, 1985.

LON, Traffic in Opium and other dangerous drugs, Analysis of the international Trade in Morphine, Diacetylmorphine, and Cocaïne for the Years 1925-1930, C.587.M.228.1930.XI.

MINISTĖRE DES AFFAIRES ETRANGÈRES, Archives, SDN, Secrétariat Général, Opium. IM.1, série 1601-1692.

MUSTO D. F., The American Disease: Origins of Narcotic Control, New York, Oxford, Oxford University Press, expanded édition, 1987.

OBSERVATOIRE GEOPOLITIQUE DES DROGUES, COUTOUZIS M., PEREZ P., Atlas Mondial des Drogues, Paris, Presses Universitaires de France, 1996.

PILA J. J., Le trafic des stupéfiants et la Société des Nations, Paris, Recueil Sirey, 1926.

PUTNAM R. D., Diplomacy and domestic Politics: the logic of two level Games, International Organizations, 42, 3, été $1988,427-460$.

RUFFAT M., 175 ans d'Industrie pharmaceutique française : histoire de Synthélabo, Paris, La découverte, 1996. 
SDN, Cartel de la cocaïne, Archive, 12/15375 (Carton R.3217).

SDN, COMITÉ CENTRAL DE L'OPIUM. Production et distribution des stupéfiants et de leurs matières premières avant la guerre, Genève, octobre 1944, C.24.M.24.1944.XI.

SDN, COMMISSION CONSULTATIVE DU TRAFIC DE L'OPIUM, Rapport de la Commission consultative du trafic de l'Opium, première session, Genève, 2-5 mai 1921, C.28.M157.1921.XI.

SDN, COMMISSION CONSULTATIVE DU TRAFIC DE L'OPIUM, Rapport au Conseil sur les travaux de la sixième session de la Commission, 4-14 août 1924.

SDN, COMMISSION CONSULTATIVE DU TRAFIC DE L'OPIUM ET AUTRES DROGUES NUISIBLES, Rapport au Conseil sur les travaux de la onzième session, Genève, 12-16 avril 1928.

SDN, COMMISSION CONSULTATIVE DU TRAFIC DE L'OPIUM ET AUTRES DROGUES NUISIBLES, Rapport au Conseil sur les travaux de la donzième session, Genève, du 17 janvier au 2 février 1929, Doc. SDN C.33.1929.XI.

SDN, COMMISSION CONSULTATIVE DU TRAFIC L'OPIUM. Exposé relatif à la fabrication de la morphine, d'autres dérivés de l'Opium et de la cocaïne, accompagné de tableaux statistiques, O.C.101.

SDN, CONFÉRENCE POUR LA LIMITATION DE LA FABRICATION DES STUPÉFIANTS, Procès verbaux révisés de la conférence, C.509.M.214.1931.XI., I. et II.

SDN, CONFÉRENCE POUR LA LIMITATION DE LA FABRICATION DES STUPÉFIANTS, Documents de travail, série Conf. L.F.S (1931).

SDN, Documents confidentiels : série O.C./Confidentiel (1933-1940).

SDN, Document de travail de la Commission du trafic de l'opium et autres drogues nuisibles : série O.C.1-O.C.1814 (1921-1942; 1943-1947).

SDN, Document de travail du Comité central permanent de l'opium: série C.C.P. 1 - C.C.P.290 (1929-1940, 1946).

SDN, Lettre de la firme Hoffman - La Roche adressée au secrétariat général de La Société des Nations le 2 juillet 1924, Archive, 14A/36370/36970 (Carton R. 787).

SDN, Procès verbal de la conférence sur la limitation de la fabrication des drogues nuisibles. Assemblée préliminaire des pays manufacturiers de drogues, Londres, octobre 1930, Archive, 12/23680/4003 (Carton; 3196-3201).

SDN, SECTION DU TRAFIC DE L'OPIUM DU SECRÉTARIAT DE LA SOCIÉTÉ DES NATIONS, Convention pour limiter la fabrication et réglementer la distribution des stupéfiants du 13 juillet 1931, Etude historique et technique, Genève, 1937.

SDN, TRAFIC DE L'OPIUM ET AUTRES DROGUES NUISIBLES, Rapport de la réunion préliminaire des représentants officiels des pays manufacturiers, tenue à Londres en octobre - novembre 1930, Genève, 1930, C.669.M.278.1930.XI.

\section{Summary}

This study has used the archives of the Society of Nations to reconsider the genesis of the international control of drugs during the period 1920-1930. It was during this period that the member states started to constrain the manufacture and international trade in drugs to medical usage, hence setting up a world-wide system of drug control which persists to today. The creation of this system involved a series of conventions which clearly distinguished legitimate and illegitimate uses of drugs. This distinction was made less at the level of the activity itself (manufacture, international trade) than in relation to those who were expressly permitted to undertake such activities. Hence the article proceeds to look at those who took part in creating the conventions and who, at the same time, were putting together a monopoly of manufacture and licit trade in drugs - states themselves and the large pharmaceutical companies.

KEY-WORdS: DRUGS - INTERNATIONAL CONTROL OF DRUGS - HISTORY OF DRUGS - INTERNATIONAL RELATIONS - MANUFACTURE AND INTERNATIONAL TRADE IN DRUGS 


\section{Zusammenfassung}

Auf der Basis von Archiven des Völkerbundes analysiert diese Untersuchung die Entstehung der internationalen Drogenkontrolle in den Jahren 1920-1930. In dieser Epoche haben sich die Staaten darauf geeinigt, die Herstellung und den internationalen Handel von Betäubungsmitteln auf medizinische Bedürfnisse zu begrenzen und so ein System der weltweiten Drogenkontrolle etabliert, das auch heute noch besteht. Die Entstehung dieses Systems manifestiert sich in einer Serie von Vereinbarungen, in denen der erlaubte und der unerlaubte Gebrauch von Drogen festgelegt worden ist. Diese Unterscheidung zwischen erlaubtem und unerlaubtem Gebrauch betrifft weniger den Gebrauch selbst (Herstellung, internationaler Handel), als vielmehr die Akteure, denen explizit der Gebrauch erlaubt ist. Deshalb bezieht sich der Artikel auf diese Akteure, die an der Ausarbeitung der Vereinbarungen beteiligt waren und die zur damaligen Zeit ein Monopol der Herstellung und des legalen Handels mit Betäubungsmitteln hatten: im wesentlichen die Staaten und große pharmazeutische Unternehmen.

\section{Samenvatting}

Tot stand gekomen op basis van de archieven van de Volkenbond, biedt deze studie aan om terug te komen op de genese van de internationale controle inzake drugs in de twintiger en dertiger jaren. Het is op dat ogenblik dat de diverse Staten mekaar vonden om de fabricatie en de internationale handel voor medische doeleinden te beperken, en dit door de installatie van een mondiaal beheerssysteem dat momenteel nog steeds in werking is. De indeplaatsstelling van dit systeem wordt geuit door een geheel van conventies die het legaal en illegaal gebruik van drugs duidelijk onderscheiden. Dit onderscheid doet zich minder voor bij de gebruikers zelf (fabricatie, internationale handel) dan wel bij de actoren die uitdrukkellijk toegelaten worden om ze te ontplooien. Dat is de reden waarom het artikel terug wil komen op de actoren die deelnamen aan de totstandkoming van de internationale conventies en die, terzelfdertijd, zich het monopolie van de fabricatie en de legale handel van drugs toeëigenden, met name de Staten en de grote pharmaceutische groepen. 